\title{
Axisymmetric fretting analysis in coated cylinder
}

\author{
M RAMESH*, SATISH V KAILAS and K R Y SIMHA \\ Department of Mechanical Engineering, Indian Institute of Science, \\ Bangalore 560012 \\ e-mail: mramesh@mecheng.iisc.ernet.in
}

\begin{abstract}
Fretting is essentially a contact fatigue phenomenon, although bulk stresses and material properties contribute to final failure. The near surface state of stress developed under oscillatory contact between machine elements plays a major role in deciding the severity of fretting. It is possible to enhance tribological properties by coating the surface. There is rather scanty literature available on fretting analysis of coated components. Presence of such coatings has a large influence on the near surface state of stress. The effect of coatings on the severity of fretting is the focus of this paper. Results obtained for both hard and soft coatings are compared with the results obtained for the homogeneous case. The component geometry and loading are chosen to be cylindrical to enable 3D elastic axisymmetric fretting analysis. The results are compared with 2D models (strip and half-plane) to examine their utility and validity for understanding axisymmetric fretting. Contact pressure and frictional shear loading cases are solved separately and superposed appropriately depending on the coefficient of friction considered. Results for different values of coefficient of friction and elastic mismatch are illustrated through contour plots of stresses and strains. These results are expected to be helpful for identifying fretting failure zones and fracture mechanisms in coated components. Analytical results presented here could serve as useful benchmarks for calibrating numerical codes and experimental techniques.
\end{abstract}

Keywords. Coated cylinder; axisymmetric analysis; static; Fourier transform.

\section{Introduction}

Fretting is essentially a contact fatigue phenomenon wherein the failure is predominantly localised near the surface (Farris et al 2003). This is indeed the case at least during the initial stages until the bulk stresses dominate and fatigue damage penetrates into the bulk material. The nature of contact between engineering components determines the near surface state of stress, which in turn controls the severity of fretting. There are cases where the engineering components are made of materials with good mechanical properties but poor tribological properties, for example, $\mathrm{Al}$ alloys used in aerospace industries. It is common to coat the substrate, or provide surface treatment. The coating material is chosen to give good tribological

*For correspondence 
properties. Such coatings exert a larger influence on the near surface state of stress. The effect of such coatings and their mechanical properties on the near surface state of stress is the focus of this paper.

There is a significant effort by metallurgists and materials scientists to synthesize new coating materials and techniques to combat corrosion and heat loss. Unfortunately, coatings are unreliable under fatigue loading in general and fretting in particular due to interfacial defects. Coatings in general can be of two types: hard and soft. Soft coatings as solid lubricants reduce the coefficient of friction though under fretting the coatings get worn out. This brings the substrate to come into contact with the pad which will lead to crack initiation. Thus, the soft coating delays crack initiation but does not prevent crack propagation. Shot peening treatment before the application of coating is found to retard crack propagation rate. However, care should be taken not to introduce cracks during the shot peening treatment. Hard coatings in general are brittle. They protect the substrate from subsequent wear and crack initiation, but they fail by delamination which in turn may even reduce fretting fatigue life. The main reason being the weak interface. Golden et al (2007); Rajasekaran et al (2008); Xu et al (2007) discussed these issues along with effects of residual stress and porosity.

Even in the absence of any coating, formation of transfer/interfacial layer between contacting parts can influence the state of stress. The coated cylinder model can also shed new light on the tribology of solid lubricants, transfer/interfacial layer, as discussed by Williams (2005). However, in the context of fretting where the amplitudes of relative motion between the contacting bodies are generally small, Hager et al (2004) reported the formation of hard tribologically transformed surface (TTS) in the mixed fretting regime where both fretting and wear are simultaneously present.

In the case of coated components fretting cracks initiated at the surface combine forces with cracks initiated at the interface to drastically reduce the fretting life. The stresses along the interface are critical to assessing the reliability of fretting components. The interfacial shear stress and the stress normal to the interface (particularly when it is tensile) control the fatigue life expectancy of coatings. Depending on the elastic mismatch between the coating and the substrate, severe stress gradients could be generated in the film.

If the coating bonds strongly with the substrate then the coating delays crack initiation and retards crack propagation as shown by experiments. Nishida et al (2003) showed experimentally that presence of lubricating anodic film delays crack initiation and also resulting in low crack growth rates. Okane et al (2003) showed that WC-Co spray over NiCrMo steel retards fretting fatigue crack initiation and lowers propagation rates. Thus, fretting fatigue strength of a component can be enhanced by coatings. Analysis of coatings in fretting fatigue theory is not emphasised in the literature. This paper is probably the first attempt aimed at a comprehensive stress analysis of coated cylinders under fretting loading.

In the context of axisymmetric cylindrical interfacial cracks Erdogan \& Ozbek (1969); Ozbek \& Erdogan (1969) followed by Farris et al (1989) and Itou (1990) analysed cylindrical interfacial cracks in infinite domain. Engineering components require solutions inside finite domain such as coated cylinders and shafts. In this class of problems before we proceed with fracture mechanics analysis, it is necessary to perform the stress analysis when the cracks are absent. This is the primary motivation for this paper. Here we show that fretting loads generate both interfacial shear and tensile stress in the coating.

Analysis of cylindrical geometries like shafts and shrink-fit assemblies generally require the application of Fourier transform technique. A comprehensive introduction to fretting analysis for a shaft with press fitted hub using Fourier transform was given by 
Dobromirsky \& Smith (1986). They simulated the effect of axial cyclic loading by superposing normal and shear stress on the cylindrical surface. They claimed that the pressure distribution at the hub shaft interface will become uniform after a few cycles due to wear of edges by fretting which otherwise would create peak stresses at the edges. They assumed as a first approximation zero shear stress in the stick region and uniform shear stress in the slip region whose magnitude depends on the coefficient of friction and the applied pressure. The above assumptions are considered valid and the same is followed in this work. Further, the contact length $2 a$, stick zone length $2 c$, contact pressure $p_{0}$ and frictional shear $q_{0}$ magnitude at the surface are all interdependent in the actual problem. However, for simplicity reasonable values of the parameters are chosen independently in this paper and the effect of each parameter on the near surface state of stress is discussed.

There is a common tendency to interpret fretting fatigue crack initiation based on indentation stress field (ISF). In reality indentation stress fields require the solution to mixed boundary problems including the possibility of stress and strain singularities at the edges. Singularities, if present in fretting, are neutralised by repeated relative motion between contacting parts. Fretting in flat and round configuration (Ciavarella \& Demelio 2001) in the context of turbine blade/disk interface present no edge singularities. The portion which sticks is mainly under Hertzian pressure loading and the shear stress effect is confined to the ends experiencing slip. The stick/slip front moves inward from the ends as the shear load builds up. Eventually, at a sufficiently high shear load, gross sliding occurs. In fretting analysis, however, we focus our attention on extremely small amplitudes of slip $(<50 \mu \mathrm{m})$ at the ends of the punch. It is assumed that the stick/slip boundary is fixed. With this idealised modelling, it is convenient to perform the stress analysis for a uniform pressure and shear profile. Thus, we reexamine and extend the work of Dobromirsky \& Smith (1986) for coated cylinders which brings in the additional complexity due to elastic mismatch.

In addition, we examine the utility of $2 \mathrm{D}$ plane strain results pertaining to a coated strip and a half-plane under identical loading. This will help us interpreting 2D fretting experiments for coated cylinders. Experimental research on fretting phenomena is generally investigated using specimens in the form of strips and plates (ASTM STP 1425). There is therefore a need to compare and correlate stresses generated by fretting loading in these three analogous geometries (cylinder, strip and half-plane, figures 1,2 and 3) to examine the utility of simplified strip and plate analysis. Comparison of stresses in strip and half-plane has already been done by Fellows et al (1995) for the homogeneous case which we now extend to the case of coated cylinder, strip and half-plane.

The coated cylinder configuration is shown in figure 1 . The configuration for coated strip and coated half-plane are shown in figures 2 and 3. The results are obtained for the following cases:

- different ratios of elastic shear moduli of coating and shaft $\left(\mu_{A} / \mu_{B}\right)$

- different values of coefficient of friction $(\phi)$

- different geometries: cylinder, strip and half-plane

- different ratios of $a / R_{1}$

- different ratios of $c / a$.

The actual fretting problem entails both pressure and shear loading which are analysed separately and superposed appropriately to simulate fretting loading. The tractions applied by Dobromirsky \& Smith (1986) comprised of uniform pressure and shear (figure 4). The same idea is followed in this paper for the case of coated cylinder subjected to fretting. 


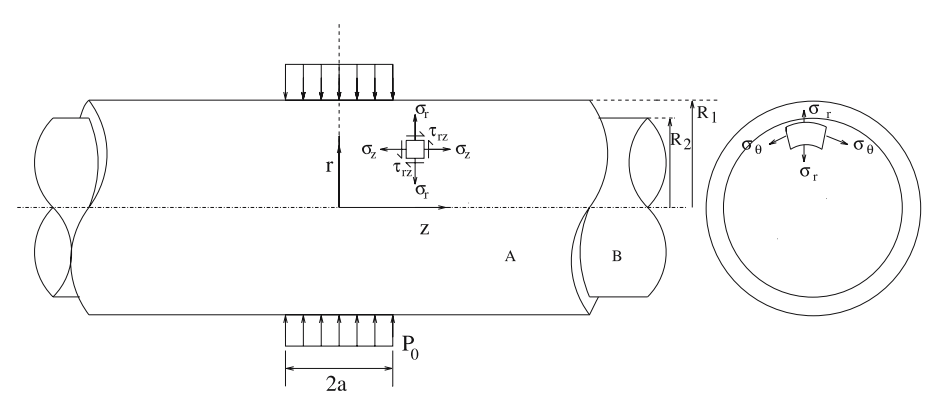

Figure 1. Coated cylinder with cylindrical co-ordinate system.

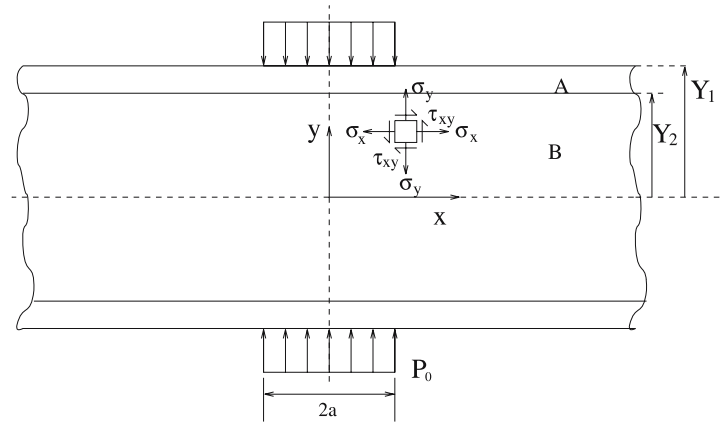

Figure 2. Coated strip with Cartesian coordinate system.

The uniform pressure/shear profile assumed in this paper provide a fundamental basis for constructing other profiles. For example, Hertzian and triangular profiles represent reality better. However, the corresponding computational complexity increases especially for coated cylinder. We therefore consider the homogeneous case separately. Another issue of some practical significance concerns the roughness of surfaces under fretting contact. Machined surfaces often exhibit regular features during the initial stages of fretting such as sinusoidal variation. The analysis for sinusoidal corrugation, for example, is possible. It is also important to bear in mind the intrinsically dynamic nature of fatigue in general and fretting in particular. This issue assumes significance when the fretting frequency lies close to the natural frequency

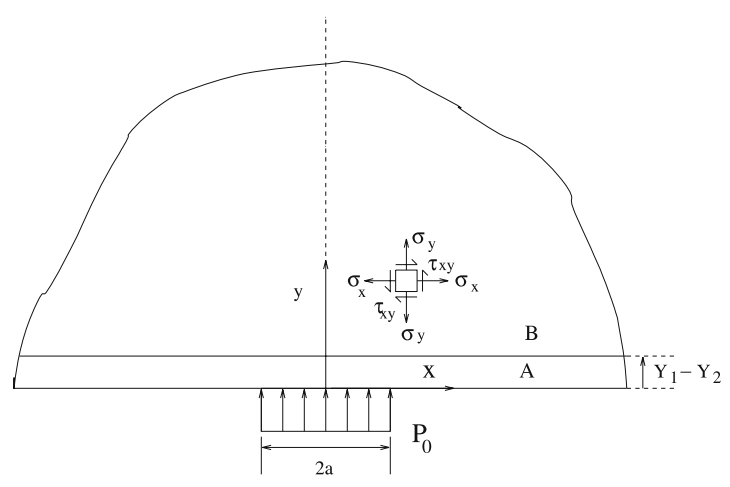

Figure 3. Coated half-plane with Cartesian co-ordinate system. 

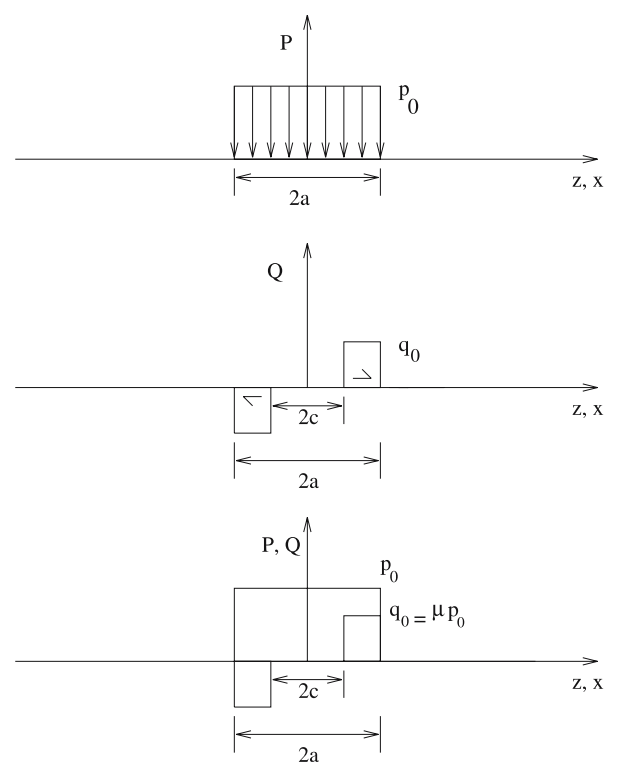

Figure 4. Traction profiles.

of the components under study. This aspect requires an advance analysis using elastodynamic theory.

\section{Formulation}

\subsection{Infinitely long coated cylinder}

Axisymmetric formulation based on Love's stress function $\Phi$ satisfies the biharmonic equation $\nabla^{2} \nabla^{2} \Phi=0$ (Timoshenko \& Goodier 1970). The method of Fourier integral transform is used to obtain the solution for the stress function, the stresses and the displacements. For more details the reader is referred to equations 1 to 16 of our paper entitled 'Near surface stress analysis strategies for Axisymetric fretting' in this issue.

In the Fourier space the biharmonic equation $\left(\nabla^{2} \nabla^{2} \Phi=0\right)$ becomes

$$
\left(\frac{d^{2}}{d r^{2}}+\frac{1}{r} \frac{d}{d r}-\xi^{2}\right)\left(\frac{d^{2} \bar{\Phi}}{d r^{2}}+\frac{1}{r} \frac{d \bar{\Phi}}{d r}-\xi^{2} \bar{\Phi}\right)=0 .
$$

The general solution to the biharmonic equation in the Fourier space is

$$
\begin{aligned}
& \bar{\Phi}_{A}=\frac{1}{\xi^{3}}\left[A_{1} \frac{I_{0}(\xi r)}{I_{0}\left(\xi R_{1}\right)}+A_{2} \frac{K_{0}(\xi r)}{K_{0}\left(\xi R_{1}\right)}+A_{3} \xi r \frac{I_{1}(\xi r)}{I_{0}\left(\xi R_{1}\right)}+A_{4} \xi r \frac{K_{1}(\xi r)}{K_{0}\left(\xi R_{1}\right)}\right] \\
& \bar{\Phi}_{B}=\frac{1}{\xi^{3}}\left[B_{1} \frac{I_{0}(\xi r)}{I_{0}\left(\xi R_{1}\right)}+B_{3} \xi r \frac{I_{1}(\xi r)}{I_{0}\left(\xi R_{1}\right)}\right],
\end{aligned}
$$

where $A_{j}(j=1,2,3,4)$ and $B_{j}(j=1,3)$ are functions of $\xi$ to be determined from boundary conditions. $I_{0}, I_{1}, K_{0}$ and $K_{1}$ are modified Bessel functions of first and second kind. Using this solution for $\bar{\Phi}_{A}$ and $\bar{\Phi}_{B}$ the stresses and displacements in the Fourier space for Cylinder $A$ (coating) and Cylinder $B$ (core) are: 
Cylinder $A$ (coating):

$$
\begin{aligned}
2 \mu_{A} \bar{u}_{r A} & =A_{1}(\xi) a_{11}(r)+A_{2}(\xi) a_{12}(r)+A_{3}(\xi) a_{13}(r)+A_{4}(\xi) a_{14}(r) \\
2 \mu_{A} \bar{u}_{z A} & =A_{1}(\xi) a_{21}(r)+A_{2}(\xi) a_{22}(r)+A_{3}(\xi) a_{23}(r)+A_{4}(\xi) a_{24}(r) \\
\bar{\sigma}_{r A} & =A_{1}(\xi) a_{31}(r)+A_{2}(\xi) a_{32}(r)+A_{3}(\xi) a_{33}(r)+A_{4}(\xi) a_{34}(r) \\
\bar{\tau}_{r z A} & =A_{1}(\xi) a_{41}(r)+A_{2}(\xi) a_{42}(r)+A_{3}(\xi) a_{43}(r)+A_{4}(\xi) a_{44}(r) \\
\bar{\sigma}_{\theta_{A}} & =A_{1}(\xi) a_{51}(r)+A_{2}(\xi) a_{52}(r)+A_{3}(\xi) a_{53}(r)+A_{4}(\xi) a_{54}(r) \\
\bar{\sigma}_{z A} & =A_{1}(\xi) a_{61}(r)+A_{2}(\xi) a_{62}(r)+A_{3}(\xi) a_{63}(r)+A_{4}(\xi) a_{64}(r) .
\end{aligned}
$$

Cylinder $B$ (core):

$$
\begin{aligned}
2 \mu_{B} \bar{u}_{r B} & =B_{1}(\xi) b_{11}(r)+B_{3}(\xi) b_{13}(r) \\
2 \mu_{B} \bar{u}_{z B} & =B_{1}(\xi) b_{21}(r)+B_{3}(\xi) b_{23}(r) \\
\bar{\sigma}_{r B} & =B_{1}(\xi) b_{31}(r)+B_{3}(\xi) b_{33}(r) \\
\bar{\tau}_{r z B} & =B_{1}(\xi) b_{41}(r)+B_{3}(\xi) b_{43}(r) \\
\bar{\sigma}_{\theta B} & =B_{1}(\xi) b_{51}(r)+B_{3}(\xi) b_{53}(r) \\
\bar{\sigma}_{z B} & =B_{1}(\xi) b_{61}(r)+B_{3}(\xi) b_{63}(r) .
\end{aligned}
$$

The expressions for $a_{i j}$ and $b_{i j}$ are given in Appendix A.1. The solution in real space can be obtained by taking the inverse Fourier transform of the above expressions. The boundary conditions for the problem at the outer radius $r=R_{1}$ are the prescribed tractions; and, at the interface radius $r=R_{2}$ stress and displacement continuity

Outer boundary: $\sigma_{r A}=P(z)$ and $\tau_{r z A}=Q(z)$

Interface: $\sigma_{r A}=\sigma_{r B} ; \tau_{r z A}=\tau_{r z B} ; u_{r A}=u_{r B} ; u_{z A}=u_{z B}$.

The above conditions in the Fourier space are given below.

Outer boundary: $\bar{\sigma}_{r A}=\bar{P} ; \bar{\tau}_{r z A}=\bar{Q}$

Interface: $\bar{\sigma}_{r A}-\bar{\sigma}_{r B}=0 ; \bar{\tau}_{r z A}-\bar{\tau}_{r z B}=0 ; \bar{u}_{r A}-\bar{u}_{r B}=0 ; \bar{u}_{z A}-\bar{u}_{z B}=0$.

The expressions for $\bar{P}$ and $\bar{Q}$ for uniform pressure and uniform double shear as in figure 4 are derived using auxiliary variable $\eta$.

Uniform pressure profile: $P(\eta)=p_{0}$ for $-a \leq \eta \leq a$

$$
\bar{P}(\xi)=\int_{0}^{\infty} P(\eta) \cos (\xi \eta) d z=\int_{0}^{a} p_{0} \cos (\xi \eta) d z=p_{0} \frac{\sin (\xi a)}{\xi} .
$$

Uniform shear profile: $Q(\eta)=q_{0}$ for $c \leq \eta \leq a$

$$
\bar{Q}(\xi)=\int_{0}^{\infty} Q(\eta) \sin (\xi \eta) d z=\int_{c}^{a} q_{0} \sin (\xi z) d z=q_{0}\left[\frac{\cos (\xi c)-\cos (\xi a)}{\xi}\right] .
$$


Also, $p_{0}$ and $q_{0}$ are related through the friction coefficient $\phi$ by $q_{0}=\phi p_{0}$. The auxiliary variable $\eta$ represents the coordinate $z$ in the axisymmetric case and $x$ in 2D plane Cartesian coordinate system.

Substituting the expressions for the transformed stresses and displacements from equations 3 and 4 in the above mentioned boundary conditions we get the following equation in the matrix form

$$
[\mathbf{M}]_{6 \times 6}[\mathbf{A}]_{6 \times 1}=[\mathbf{P}]_{6 \times 1}
$$

where,

$$
\begin{aligned}
& \mathbf{M}=\left[\begin{array}{cccccc}
a_{31}\left(R_{1}\right) & a_{32}\left(R_{1}\right) & a_{33}\left(R_{1}\right) & a_{34}\left(R_{1}\right) & 0 & 0 \\
a_{41}\left(R_{1}\right) & a_{42}\left(R_{1}\right) & a_{43}\left(R_{1}\right) & a_{44}\left(R_{1}\right) & 0 & 0 \\
a_{31}\left(R_{2}\right) & a_{32}\left(R_{2}\right) & a_{33}\left(R_{2}\right) & a_{34}\left(R_{2}\right) & -b_{31}\left(R_{2}\right) & -b_{33}\left(R_{2}\right) \\
a_{41}\left(R_{2}\right) & a_{42}\left(R_{2}\right) & a_{43}\left(R_{2}\right) & a_{44}\left(R_{2}\right) & -b_{41}\left(R_{2}\right) & -b_{43}\left(R_{2}\right) \\
a_{11}\left(R_{2}\right) & a_{12}\left(R_{2}\right) & a_{13}\left(R_{2}\right) & a_{14}\left(R_{2}\right) & -\frac{\mu_{A}}{\mu_{B}} b_{11}\left(R_{2}\right) & -\frac{\mu_{A}}{\mu_{B}} b_{13}\left(R_{2}\right) \\
a_{21}\left(R_{2}\right) & a_{22}\left(R_{2}\right) & a_{23}\left(R_{2}\right) & a_{24}\left(R_{2}\right) & -\frac{\mu_{A}}{\mu_{B}} b_{21}\left(R_{2}\right) & -\frac{\mu_{A}}{\mu_{B}} b_{23}\left(R_{2}\right)
\end{array}\right] \\
& \mathbf{A}=\left[\begin{array}{c}
A_{1} \\
A_{2} \\
A_{3} \\
A_{4} \\
B_{1} \\
B_{3}
\end{array}\right] ; \quad \mathbf{P}=\left[\begin{array}{c}
\bar{P} \\
\bar{Q} \\
0 \\
0 \\
0 \\
0
\end{array}\right]
\end{aligned}
$$

The problems of pressure $(\bar{Q}=0)$ and shear loading $(\bar{P}=0)$ are solved separately. The results are superposed appropriately to obtain the full solution. The six unknowns $A_{1}(\xi), A_{2}(\xi), A_{3}(\xi), A_{4}(\xi), B_{1}(\xi)$ and $B_{3}(\xi)$ are determined by solving the above matrix equation. Substituting these functions and inverting equations 3 and 4 , the stresses and displacements are obtained. The formulation discussed in this paper corresponds to coated solid cylinders. Since hollow cylinders are often used in applications the formulation can be easily extended to a coated hollow cylinder by taking

$$
\bar{\Phi}_{B}=\frac{1}{\xi^{3}}\left[B_{1} \frac{I_{0}(\xi r)}{I_{0}\left(\xi R_{1}\right)}+B_{2} \frac{K_{0}(\xi r)}{K_{0}\left(\xi R_{1}\right)}+B_{3} \xi r \frac{I_{1}(\xi r)}{I_{0}\left(\xi R_{1}\right)}+B_{4} \xi r \frac{K_{1}(\xi r)}{K_{0}\left(\xi R_{1}\right)}\right]
$$

with two more extra boundary conditions along the inner boundary of the cylinder.

Owing to the axisymmetric nature, the tangential stress $\sigma_{\theta}$ becomes a principal stress. The other two principal stresses are

$$
\frac{\sigma_{r}+\sigma_{z}}{2} \pm\left[\left(\frac{\sigma_{r}-\sigma_{z}}{2}\right)^{2}+\tau_{r z}^{2}\right] .
$$

Algebraically sorting these three values gives $\sigma_{1}, \sigma_{2}$ and $\sigma_{3}$ from which $\tau_{o c t}$ can be obtained. 


\subsection{Coated strip and half-plane}

The formulation for strip and half-plane is done using plane Airy's stress function in conjunction with Fourier transforms. The procedure followed is same as that of cylindrical case. It should be noted that Fourier cosine transforms are required for symmetric loading. The main results are listed below. The Airy stress function in the Fourier space for coated strip and coated half-plane are as follows.

The Airy stress functions for the coating $A$ and the strip $B$ (figure 2) are:

$$
\begin{aligned}
& \bar{\Phi}_{A}=\frac{1}{\xi^{2}}\left[A_{1} e^{-\xi\left(y-Y_{1}\right)}+A_{2} e^{\xi\left(y-Y_{1}\right)}+A_{3} \xi y e^{-\xi\left(y-Y_{1}\right)}+A_{4} \xi y e^{\xi\left(y-Y_{1}\right)}\right] \\
& \bar{\Phi}_{B}=\frac{1}{\xi^{2}}\left[B_{1} \cosh (\xi y) / e^{-\xi Y_{1}}+B_{3} \xi y \sinh (\xi y) / e^{-\xi Y_{1}}\right] .
\end{aligned}
$$

The Airy stress functions for the coating $A$ and the half-plane $B$ (figure 3 ) are

$$
\begin{aligned}
& \bar{\Phi}_{A}=\frac{1}{\xi^{2}}\left[A_{1} e^{-\xi y}+A_{2} e^{\xi y}+A_{3} \xi y e^{-\xi y}+A_{4} \xi y e^{\xi y}\right] \\
& \bar{\Phi}_{B}=\frac{1}{\xi^{2}}\left[B_{1} e^{-\xi y}+B_{3} \xi y e^{-\xi y}\right] .
\end{aligned}
$$

The stresses and displacements in the Fourier space are:

Coating $A$ :

$$
\begin{aligned}
2 \mu_{A} \bar{u}_{y A} & =A_{1}(\xi) a_{11}(y)+A_{2}(\xi) a_{12}(y)+A_{3}(\xi) a_{13}(y)+A_{4}(\xi) a_{14}(y) \\
2 \mu_{A} \bar{u}_{x A} & =A_{1}(\xi) a_{21}(y)+A_{2}(\xi) a_{22}(y)+A_{3}(\xi) a_{23}(y)+A_{4}(\xi) a_{24}(y) \\
\bar{\sigma}_{y A} & =A_{1}(\xi) a_{31}(y)+A_{2}(\xi) a_{32}(y)+A_{3}(\xi) a_{33}(y)+A_{4}(\xi) a_{34}(y) \\
\bar{\tau}_{x y A} & =A_{1}(\xi) a_{41}(y)+A_{2}(\xi) a_{42}(y)+A_{3}(\xi) a_{43}(y)+A_{4}(\xi) a_{44}(y) \\
\bar{\sigma}_{w_{A}} & =A_{1}(\xi) a_{51}(y)+A_{2}(\xi) a_{52}(y)+A_{3}(\xi) a_{53}(y)+A_{4}(\xi) a_{54}(y) \\
\bar{\sigma}_{x A} & =A_{1}(\xi) a_{61}(y)+A_{2}(\xi) a_{62}(y)+A_{3}(\xi) a_{63}(y)+A_{4}(\xi) a_{64}(y) .
\end{aligned}
$$

Strip/half-plane $B$ :

$$
\begin{aligned}
2 \mu_{B} \bar{u}_{y B} & =B_{1}(\xi) b_{11}(y)+B_{3}(\xi) b_{13}(y) \\
2 \mu_{B} \bar{u}_{x B} & =B_{1}(\xi) b_{21}(y)+B_{3}(\xi) b_{23}(y) \\
\bar{\sigma}_{y B} & =B_{1}(\xi) b_{31}(y)+B_{3}(\xi) b_{33}(y) \\
\bar{\tau}_{x y B} & =B_{1}(\xi) b_{41}(y)+B_{3}(\xi) b_{43}(y) \\
\bar{\sigma}_{w B} & =B_{1}(\xi) b_{51}(y)+B_{3}(\xi) b_{53}(y) \\
\bar{\sigma}_{x B} & =B_{1}(\xi) b_{61}(y)+B_{3}(\xi) b_{63}(y) .
\end{aligned}
$$


The corresponding expressions for $a_{i j}$ and $b_{i j}$ for strip and half-plane are given in the Appendix A.2 and A.3. The boundary conditions in the Fourier space are:

Outer boundary: $\bar{\sigma}_{y A}=\bar{P} ; \bar{\tau}_{x y A}=\bar{Q}$.

Interface: $\bar{\sigma}_{y A}-\bar{\sigma}_{y B}=0 ; \bar{\tau}_{x y A}-\bar{\tau}_{x y B}=0 ; \bar{u}_{y A}-\bar{u}_{y B}=0 ; \bar{u}_{x A}-\bar{u}_{x B}=0$.

\section{Results and discussion}

An infinitely long coated cylinder with coating $A$ and core $B$ is considered for analysis with $R_{1}$ and $R_{2}$ being the outer and interface radii. The width of pressure band is taken as $a=0 \cdot 15 R_{1}$. The width of the slip region is taken equal to the coating thickness $c=a / 2$. The interface radius $R_{2}=R_{1}-c$. The coating thickness is generally of the order ranging from few microns to a fraction of $\mathrm{mm}$. In this analysis the coating thickness is equal to half of the semi contact length. Both hard and soft coatings are considered. Four different elastic mismatch ratios considered are $\mu_{A} / \mu_{B}=0.5,1,2,5$ with the Poisson's ratio $(v=0.25)$ being same for both coating and substrate. Suffixes $A$ and $B$ are used to represent coating and substrate, respectively. The cases of $\mu_{A}=2 \mu_{B}, 5 \mu_{B}$ correspond to the case of hard coating and $\mu_{A}=\frac{\mu_{B}}{2}$ corresponds to the case of soft coating. The case of $\mu_{A}=\mu_{B}$ corresponds to a homogeneous cylinder which is considered as the standard with which other cases can be compared. The coefficients of friction $\phi$ are $0,0.3,0.7$ and $\infty$ (pure shear). The stresses obtained from cylinder are compared with the stresses obtained from $2 \mathrm{D}$ models for particular values of $\mu$ and $\phi$. Different values of the ratios $a / R_{1}$ and $c / a$ are considered for illustrating the results.

The matrix equation 7 is solved numerically and the arbitrary constants $A_{1}$ to $A_{4}, B_{1}$ and $B_{3}$ are obtained. The stress values have been evaluated by substituting the values obtained for the arbitrary constants and by taking the corresponding inverse transforms of equations 3 and 4 . The actual values of Bessel functions are used for calculating $a_{i j}$ and $b_{i j}$. The range of $\xi$ is taken from $1 \times 10^{-20}$ to 350 for implementing numerical calculations. Beyond 350 asymptotic expressions are used up to 1050. For evaluating the integrals of inverse transforms, trapezoidal rule is used with the interval of $\xi$ taken to be unity.

Similarly for the cases of strip and half-plane inversion of equations 12 to 13 gives the stresses and displacements. For numerical inversion actual values of hyperbolic and exponential functions are used for $\xi=1 \times 10^{-20}$ to 700 with the interval for numerical integration by trapezoidal rule taken as unity.

We present the results in the vicinity of the edge of the loading zone in a rectangular window $2 a \times a$. It is a common practice to normalise stress with respect to the applied contact pressure. In the case of coated components, however, it is also advantageous to examine strains. In fretting contact problems the stress gradients near the contact are generally high. Owing to this feature, crack initiation appears to depend on the state of stress developed in a characteristic volume of material rather than a single material point on the surface, (Nowell \& Araujo 2003). Hence contour plots will reveal the areas over which high stress gradients are present which can be used for multi-axial fatigue modelling (Papadopoulos et al 1997; Goncalves et al 2005) to predict crack initiation. The contours of positive and negative values are shown in red and blue colours. The contours corresponding to zero and near zero values are shown in black. The green solid line shows the interface between the coating and the substrate whereas the dotted green line in homogeneous solid shows the position of interface which is present in the coated case. The contours of different stresses and strains normalised with the applied pressure are presented in the range of -3 to 3 with $0 \cdot 1$ interval. 
Depending on the elastic mismatch between the coating and the substrate, the stresses in the substrate need to be rescaled to be seen in the contour plots. High stresses are induced in hard coatings while soft coatings are stressed little. It is therefore convenient to rescale the stresses in the substrate by the ratio of moduli for stress components that are discontinuous across the coating like $\sigma_{z}$ and $\sigma_{\theta}$ (see Appendix B for scaling factors). The other two stresses are not rescaled. Similarly, the strain $\epsilon_{r}$ and $\tau_{o c t}$ are rescaled. It should be noted that rescaling reveals more detailed information.

\subsection{Effect of elastic mismatch}

The coating applied to the surface can be either soft or hard compared to the substrate. They are found to respond to the fretting tractions differently. Soft coatings are generally thick and hence if the interface is not sufficiently strong delamination can occur (Xu et al 2007). In contrast, hard coatings are generally thin and may fail in a brittle manner when subjected to fatigue loading (Golden et al 2007). Thus the mechanism of failure of soft and hard coatings are different and hence it is important to study the effect of elastic mismatch. In this analysis the Poisson's ratio of the substrate and coating are assumed to be same and only the effect of different shear moduli of coating and substrate is discussed.

Figure 5 shows contours of $\sigma_{r}$ in the $2 a \times a$ window with its center coinciding under the end of the pressure band along the interface. The coefficient of friction is taken as $\phi=0.7$ and four different elastic mismatch as mentioned earlier are considered. In general, coefficient of friction is generally found to be high in fretting fatigue contact and hence a high value of 0.7 is chosen (Dobromirsky \& Smith 1986). The figure shows that for different elastic mismatches the contours of $\sigma_{r}$ look similar with only minor changes. Similar result is shown for the $\tau_{r z}$ in figure 6 . Also it can be seen that these stresses are continuous along the interface as demanded by the boundary condition.

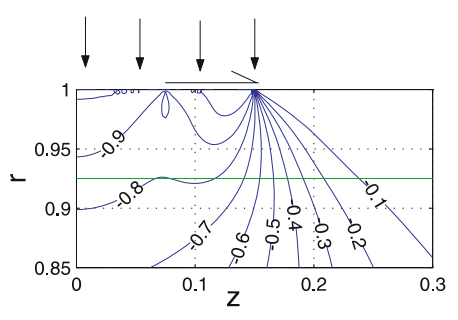

(a) $\sigma_{\mathrm{r}} / \mathrm{p}_{0}\left(\mu_{\mathrm{A}}=0.5 \mu_{\mathrm{B}}\right)$

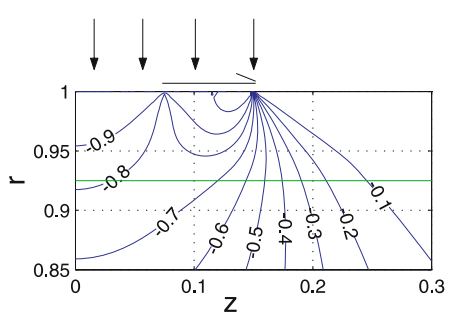

(c) $\sigma_{\mathrm{r}} / \mathrm{p}_{0}\left(\mu_{\mathrm{A}}=2 \mu_{\mathrm{B}}\right)$

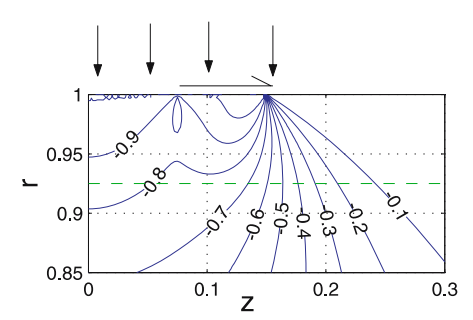

(b) $\sigma_{\mathrm{r}} / \mathrm{p}_{0}\left(\mu_{\mathrm{A}}=\mu_{\mathrm{B}}\right)$

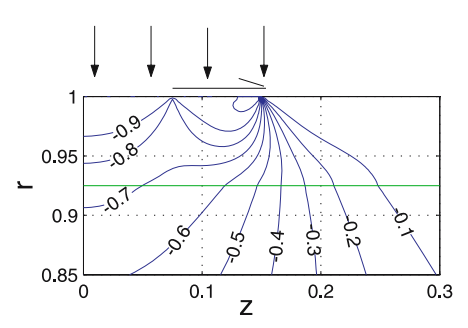

(d) $\sigma_{\mathrm{r}} / \mathrm{p}_{0}\left(\mu_{\mathrm{A}}=5 \mu_{\mathrm{B}}\right)$

Figure 5. Contours of $\sigma_{r}$ in cylinder $\left(\phi=0 \cdot 7, a=0 \cdot 15 R_{1}\right.$ and $\left.c=0 \cdot 5 a\right)$. 


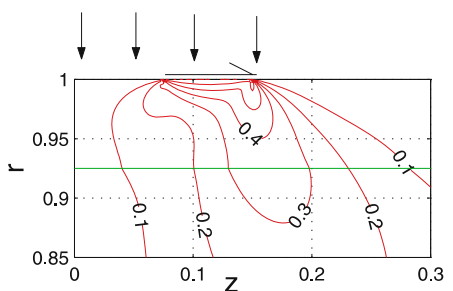

(a) $\tau_{\mathrm{rz}} / \mathrm{p}_{0}\left(\mu_{\mathrm{A}}=0.5 \mu_{\mathrm{B}}\right)$

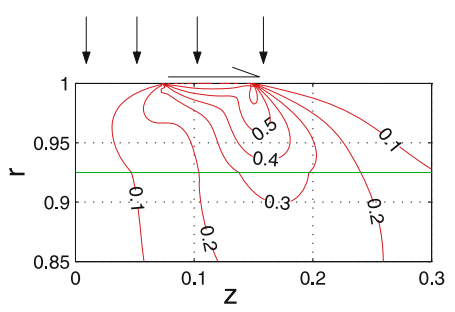

(c) $\tau_{\mathrm{rz}} / \mathrm{p}_{0}\left(\mu_{\mathrm{A}}=2 \mu_{\mathrm{B}}\right)$

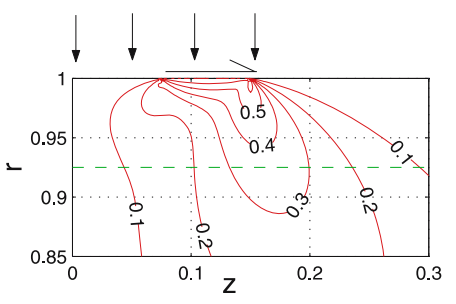

(b) $\tau_{\mathrm{rz}} / \mathrm{p}_{0}\left(\mu_{\mathrm{A}}=\mu_{\mathrm{B}}\right)$

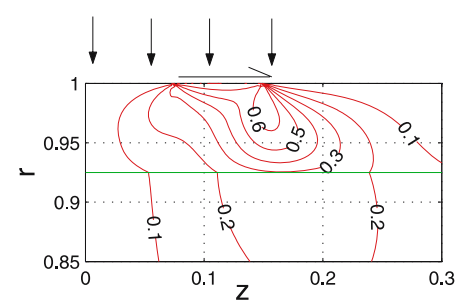

(d) $\tau_{\mathrm{rz}} / \mathrm{p}_{0}\left(\mu_{\mathrm{A}}=5 \mu_{\mathrm{B}}\right)$

Figure 6. Contours of $\tau_{r z}$ in cylinder $\left(\phi=0.7, a=0.15 R_{1}\right.$ and $\left.c=0.5 a\right)$.

However for the case of $\sigma_{z}$, the elastic mismatch has a larger effect as seen from figure 7. It can be shown that as the coating becomes much harder than the substrate, $\sigma_{z}$ becomes tensile in a region nearer to the interface. This shows the bending of the coating due to normal pressure as the core becomes comparatively softer. For the case of $\sigma_{\theta}$ (figure 8) the stress component remains compressive even with increasing elastic mismatch. It should be remembered that stresses in the substrate are rescaled with the ratio of elastic mismatch for the cases of $\sigma_{z}$ and $\sigma_{\theta}$. These stresses are discontinuous along the interface as expected.

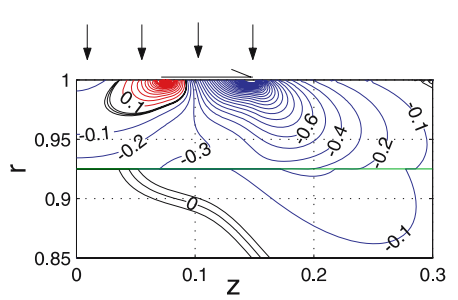

(a) $\sigma_{z} / p_{0}\left(\mu_{\mathrm{A}}=0.5 \mu_{\mathrm{B}}\right)$

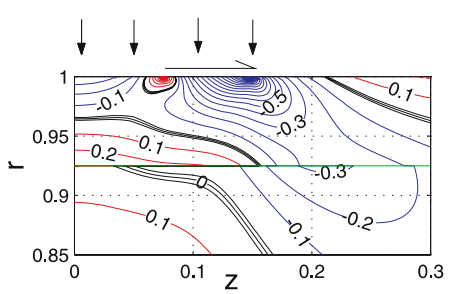

(c) $\sigma_{\mathrm{z}} / \mathrm{p}_{0}\left(\mu_{\mathrm{A}}=2 \mu_{\mathrm{B}}\right)$

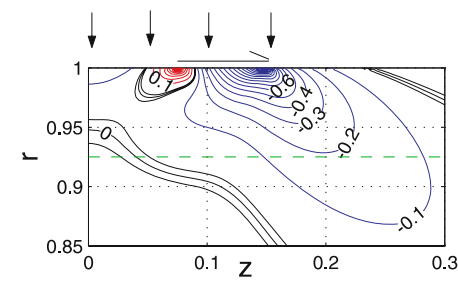

(b) $\sigma_{z} / p_{0}\left(\mu_{\mathrm{A}}=\mu_{\mathrm{B}}\right)$

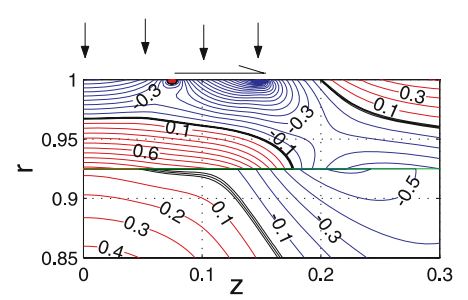

(d) $\sigma_{z} / p_{0}\left(\mu_{\mathrm{A}}=5 \mu_{\mathrm{B}}\right)$

Figure 7. Contours of $\sigma_{z}$ in cylinder ( $\phi=0 \cdot 7, a=0 \cdot 15 R_{1}$ and $\left.c=0 \cdot 5 a\right)$. 


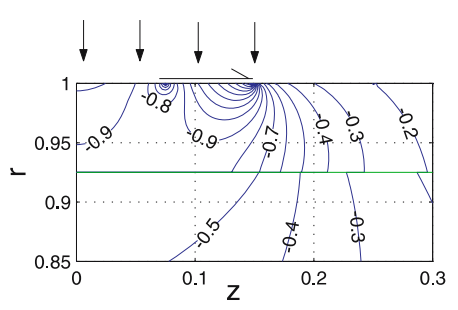

(a) $\sigma_{\theta} / \mathrm{p}_{0}\left(\mu_{\mathrm{A}}=0.5 \mu_{\mathrm{B}}\right)$

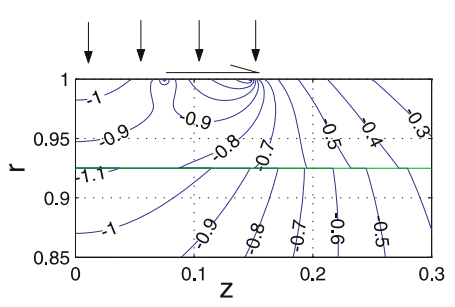

(c) $\sigma_{\theta} / p_{0}\left(\mu_{\mathrm{A}}=2 \mu_{\mathrm{B}}\right)$

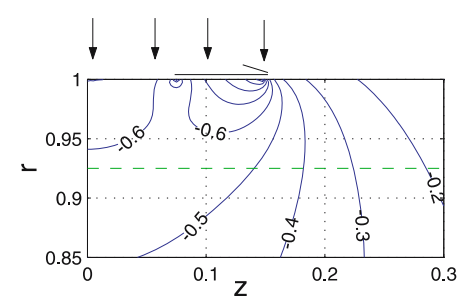

(b) $\sigma_{\theta} / p_{0}\left(\mu_{\mathrm{A}}=\mu_{\mathrm{B}}\right)$

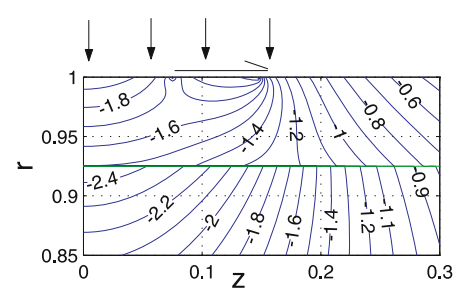

(d) $\sigma_{\theta} / p_{0}\left(\mu_{\mathrm{A}}=5 \mu_{\mathrm{B}}\right)$

Figure 8. Contours of $\sigma_{\theta}$ in cylinder $\left(\phi=0 \cdot 7, a=0.15 R_{1}\right.$ and $\left.c=0.5 a\right)$.

The contours of $\tau_{o c t}$ which controls plasticity and potential sites for crack initiation are shown in figure 9. The figure shows that contours look similar for homogeneous and soft coating case. However, the contours change shape significantly for the case of hard coating. The contours in the core are plotted after rescaling with the corresponding modulus ratio. Figure 10 shows the contours of dilatation where we see that it remains compressive for all the four cases of elastic mismatch with the magnitudes increasing with increasing coating

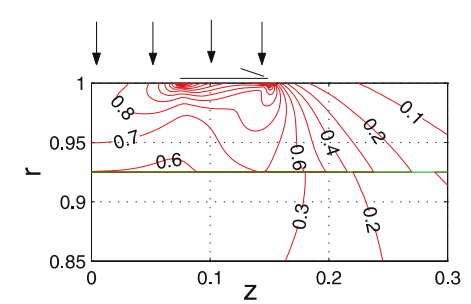

(a) $\tau_{\text {oct }} / p_{0}\left(\mu_{\mathrm{A}}=0.5 \mu_{\mathrm{B}}\right)$

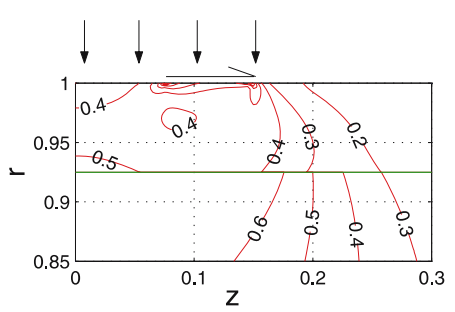

(c) $\tau_{\text {oct }} / \mathrm{p}_{0}\left(\mu_{\mathrm{A}}=2 \mu_{\mathrm{B}}\right)$

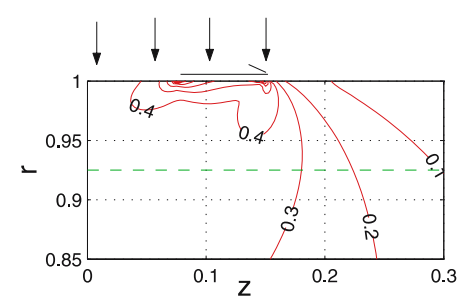

(b) $\tau_{\text {oct }} / p_{0}\left(\mu_{\mathrm{A}}=\mu_{\mathrm{B}}\right)$

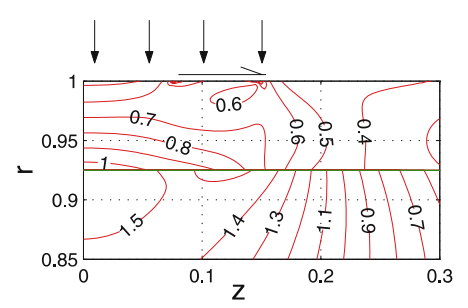

(d) $\tau_{\text {oct }} / p_{0}\left(\mu_{\mathrm{A}}=5 \mu_{\mathrm{B}}\right)$

Figure 9. Contours of $\tau_{o c t}$ in cylinder $\left(\phi=0 \cdot 7, a=0.15 R_{1}\right.$ and $\left.c=0.5 a\right)$. 


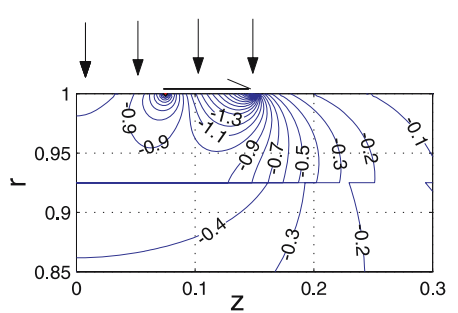

(a) $\Delta / \mathrm{p}_{0}\left(\mu_{\mathrm{A}}=0.5 \mu_{\mathrm{B}}\right)$

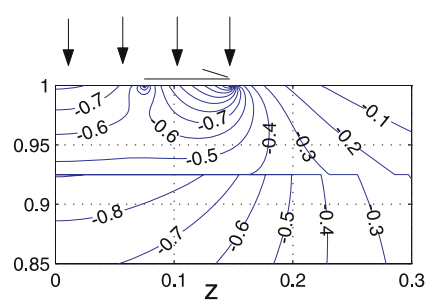

(c) $\Delta / \mathrm{p}_{0}\left(\mu_{\mathrm{A}}=2 \mu_{\mathrm{B}}\right)$

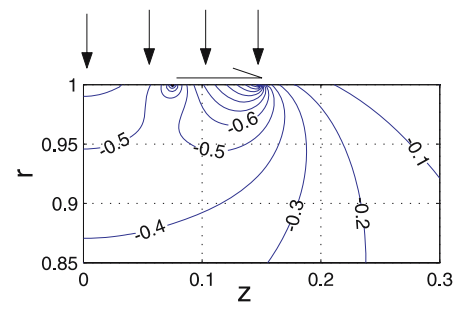

(b) $\Delta / \mathrm{p}_{0}\left(\mu_{\mathrm{A}}=\mu_{\mathrm{B}}\right)$

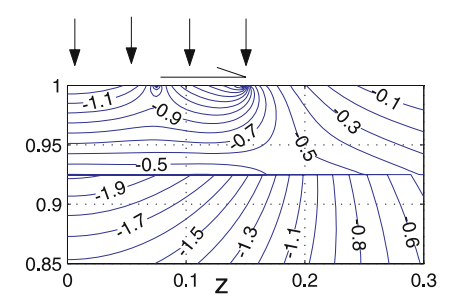

(d) $\Delta / p_{0}\left(\mu_{\mathrm{A}}=5 \mu_{\mathrm{B}}\right)$

Figure 10. Contours of $\Delta$ in cylinder $\left(\phi=0 \cdot 7, a=0.15 R_{1}\right.$ and $\left.c=0.5 a\right)$.

hardness. The contours of strain components $\epsilon_{r}$ and $\epsilon_{z}$ are shown in figures 11 and 12. The $\epsilon_{r}$ in the core cylinder is rescaled with ratio of elastic shear modulus and $\epsilon_{z}$ is not rescaled since it is continuous at the interface. The $\epsilon_{r}$ in soft coating is higher than homogeneous case and it decreases with increasing $\mu_{A}$ elastic shear modulus of the coating. This shows that soft coating is more compliant and would take any plastic deformation due to contact load without deteriorating the substrate. But hard coatings are less compliant and more brittle which may lead to failure of coating especially when they are subjected to fatigue loading.

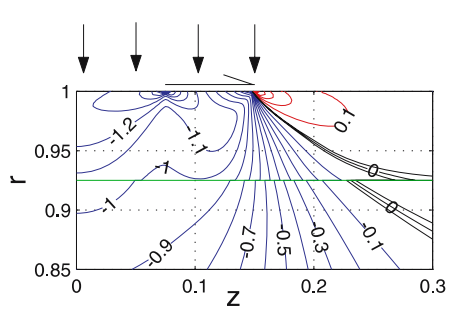

(a) $\mathrm{E} \varepsilon_{\mathrm{r}} / \mathrm{p}_{0}\left(\mu_{\mathrm{A}}=0.5 \mu_{\mathrm{B}}\right)$

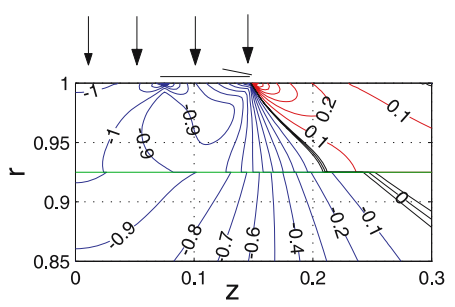

(c) $\mathrm{E} \varepsilon_{\mathrm{r}} / \mathrm{p}_{0}\left(\mu_{\mathrm{A}}=2 \mu_{\mathrm{B}}\right)$

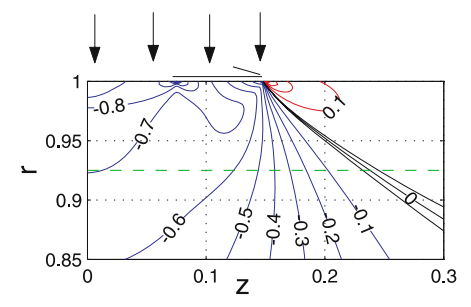

(b) $\mathrm{E} \varepsilon_{\mathrm{r}} / \mathrm{p}_{0}\left(\mu_{\mathrm{A}}=\mu_{\mathrm{B}}\right)$

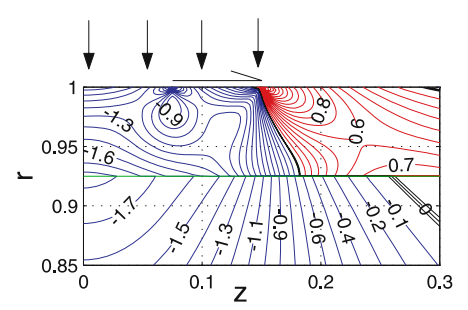

(d) $\mathrm{E} \varepsilon_{\mathrm{r}} / \mathrm{p}_{0}\left(\mu_{\mathrm{A}}=5 \mu_{\mathrm{B}}\right)$

Figure 11. Contours of $\epsilon_{r}$ in cylinder ( $\phi=0.7, a=0.15 R_{1}$ and $\left.c=0.5 a\right)$. 


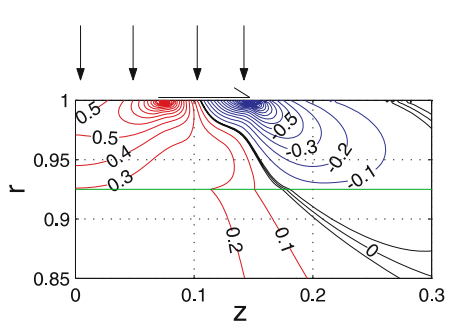

(a) $\mathrm{E} \varepsilon_{\mathrm{z}} / \mathrm{p}_{0}\left(\mu_{\mathrm{A}}=0.5 \mu_{\mathrm{B}}\right)$

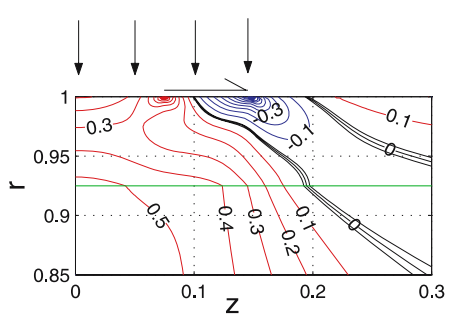

(c) $\mathrm{E} \varepsilon_{z} / \mathrm{p}_{0}\left(\mu_{\mathrm{A}}=2 \mu_{\mathrm{B}}\right)$

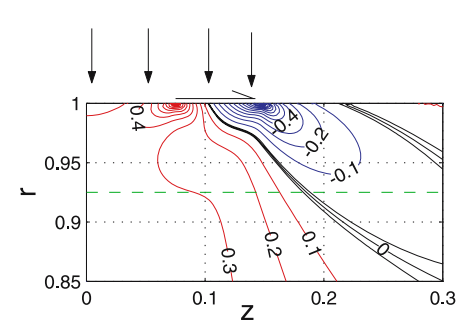

(b) $\mathrm{E} \varepsilon_{\mathrm{z}} / \mathrm{p}_{0}\left(\mu_{\mathrm{A}}=\mu_{\mathrm{B}}\right)$

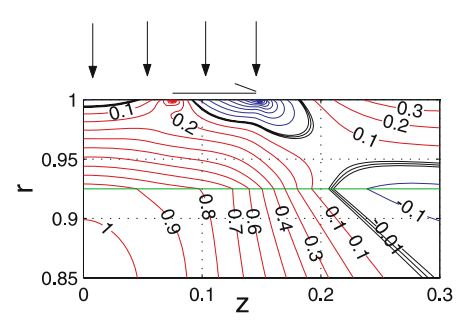

(d) $\mathrm{E} \varepsilon_{\mathrm{z}} / \mathrm{p}_{0}\left(\mu_{\mathrm{A}}=5 \mu_{\mathrm{B}}\right)$

Figure 12. Contours of $\epsilon_{z}$ in cylinder $\left(\phi=0 \cdot 7, a=0 \cdot 15 R_{1}\right.$ and $\left.c=0.5 a\right)$.

Again the value of $\epsilon_{z}$ is highest for hard coating. This could be attributed to the bending of the coating in presence of comparatively soft substrate. In the above discussion the hardness is correlated with the elastic shear modulus $(\mu)$ since they are generally proportional.

\subsection{Effect of coefficient of friction}

The best way to reduce the severity of fretting is to reduce the coefficient of friction $\phi$. The coatings are generally expected to reduce the coefficient of friction. The hardness of the

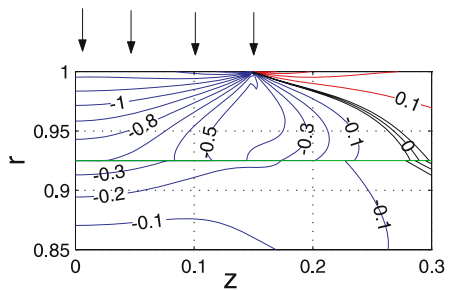

(a) $\sigma_{z} / p_{0}(\phi=0)$

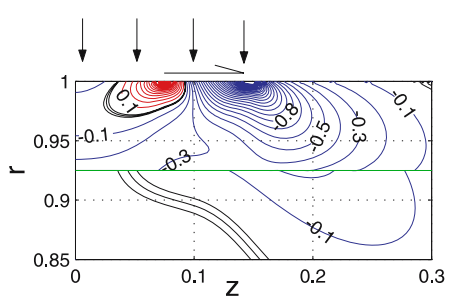

(c) $\sigma_{z} / p_{0}(\phi=0.5)$

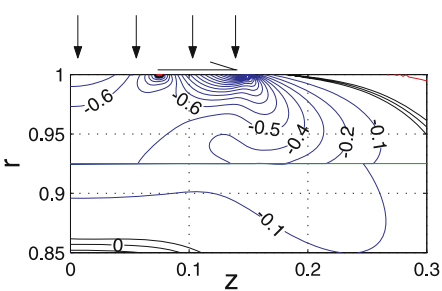

(b) $\sigma_{\mathrm{z}} / \mathrm{p}_{0}(\phi=0.3)$

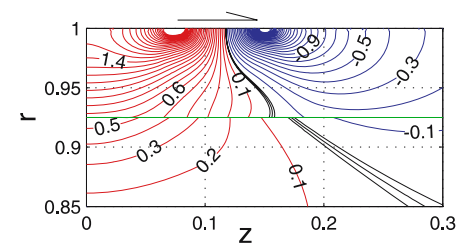

(d) $\sigma_{z} / p_{0}$ (pure shear)

Figure 13. Contours of $\sigma_{z}$ in cylinder $\left(\mu_{A}=0 \cdot 5 \mu_{B}, a=0 \cdot 15 R_{1}\right.$ and $\left.c=0 \cdot 5 a\right)$. 


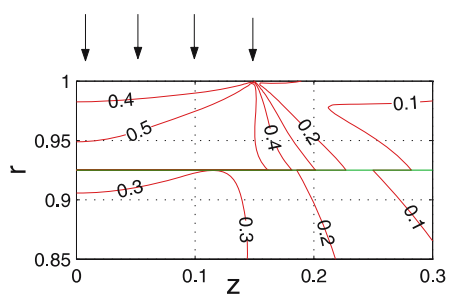

(a) $\tau_{\text {oct }} / \mathrm{p}_{0}(\phi=0)$

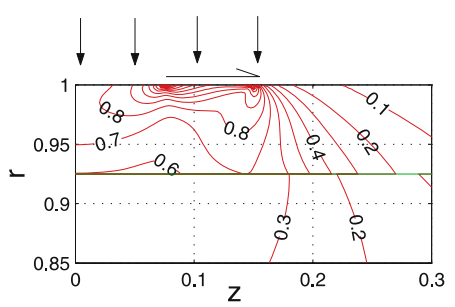

(c) $\tau_{\text {oct }} / \mathrm{p}_{0}(\phi=0.5)$

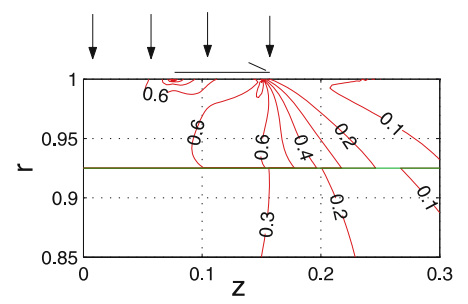

(b) $\tau_{\text {oct }} / p_{0}(\phi=0.3)$

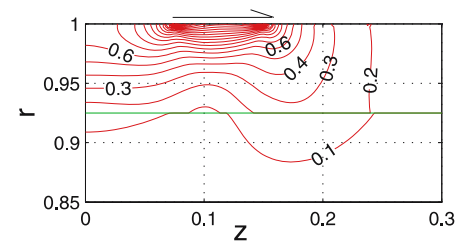

(d) $\tau_{\text {oct }} / \mathrm{p}_{0}$ (pure shear)

Figure 14. Contours of $\tau_{o c t}$ in cylinder $\left(\mu_{A}=0.5 \mu_{B}, a=0.15 R_{1}\right.$ and $\left.c=0.5 a\right)$.

coating also plays a role. Hence the effect of friction is studied here with reference to hard and soft coating separately. Fretting problem always lies between the two extremes of only pressure (figure 13a) and only shear loading (figure 13d) cases. Hence two different cases of coefficient of friction $(\phi=0.3,0.7)$ are studied along with the cases of only pressure $(\phi=0)$ and only shear (without any pressure applied on the surface). The high friction coefficient can be reduced by using solid lubricants. Hence a lower value of 0.3 is also used in the analysis.

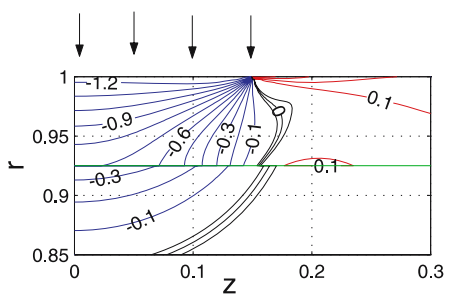

(a) $\sigma_{1} / \mathrm{p}_{0}(\phi=0)$

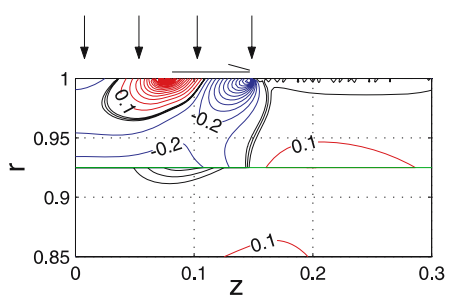

(c) $\sigma_{1} / \mathrm{p}_{0}(\phi=0.5)$

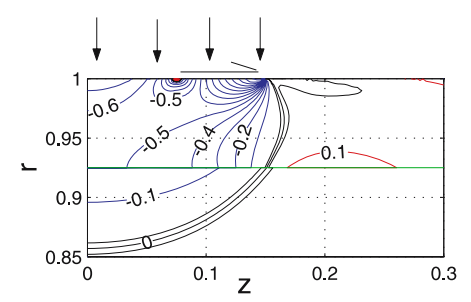

(b) $\sigma_{1} / p_{0}(\phi=0.3)$

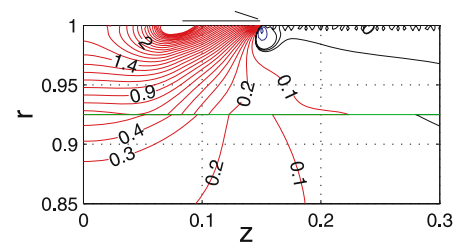

(d) $\sigma_{1} / p_{0}$ (pure shear)

Figure 15. Contours of $\sigma_{1}$ in cylinder $\left(\mu_{A}=0 \cdot 5 \mu_{B}, a=0 \cdot 15 R_{1}\right.$ and $\left.c=0.5 a\right)$. 


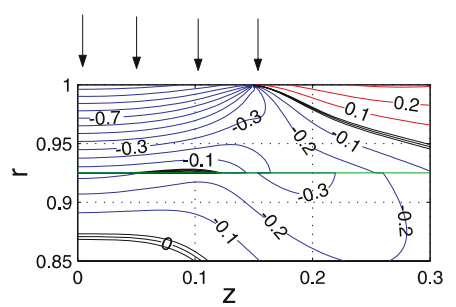

(a) $\sigma_{z} / p_{0}(\phi=0)$

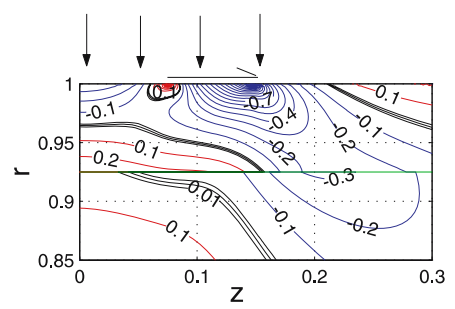

(c) $\sigma_{\mathrm{z}} / \mathrm{p}_{0}(\phi=0.5)$

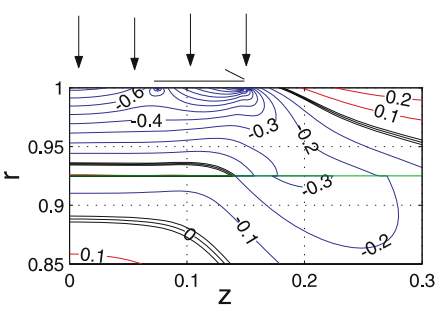

(b) $\sigma_{z} / \mathrm{p}_{0}(\phi=0.3)$

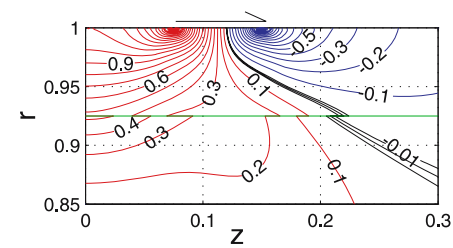

(d) $\sigma_{z} / p_{0}$ (pure shear)

Figure 16. Contours of $\sigma_{z}$ in cylinder $\left(\mu_{A}=2 \mu_{B}, a=0 \cdot 15 R_{1}\right.$ and $\left.c=0 \cdot 5 a\right)$.

Figure 13 shows contours of $\sigma_{z}$ for the case of soft coating $\mu_{A}=0.5 \mu_{B}$. This shows as the coefficient of friction is increased the $\sigma_{z}$ becomes tensile starting from the trailing edge of the shear profile and hence is important.

For soft coating the contours of $\tau_{o c t}$ and $\sigma_{1}$ are shown in figures 14 and 15 . It can be seen that as $\phi$ increases the stresses become more concentrated at the surface and $\sigma_{1}$ becomes tensile similar to $\sigma_{z}$.

Similar results are shown in figures 16,17 and 18 for the case of hard coating $\left(\mu_{A}=2 \mu_{B}\right)$. This shows that as the coefficient of friction increases the magnitude of shear loading leading to further concentration of the stresses in the coating.

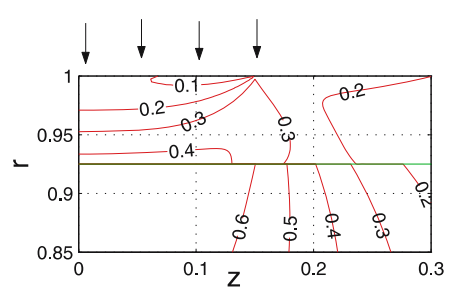

(a) $\tau_{\text {oct }} / \mathrm{p}_{0}(\phi=0)$

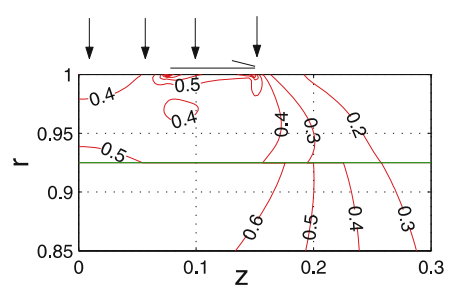

(c) $\tau_{\text {oct }} / \mathrm{p}_{0}(\phi=0.5)$

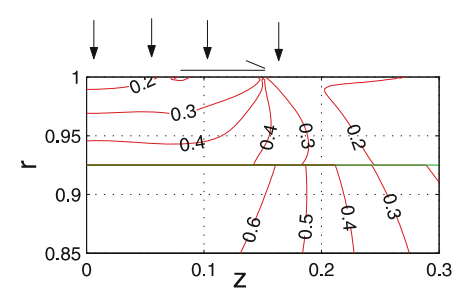

(b) $\tau_{\text {oct }} / \mathrm{p}_{0}(\phi=0.3)$

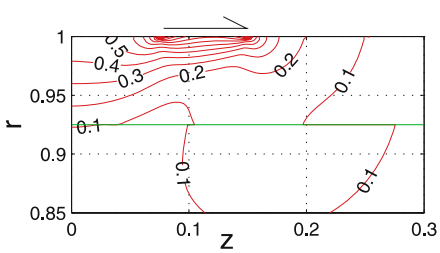

(d) $\tau_{\text {oct }} / p_{0}$ (pure shear)

Figure 17. Contours of $\tau_{o c t}$ in cylinder $\left(\mu_{A}=2 \mu_{B}, a=0 \cdot 15 R_{1}\right.$ and $\left.c=0 \cdot 5 a\right)$. 


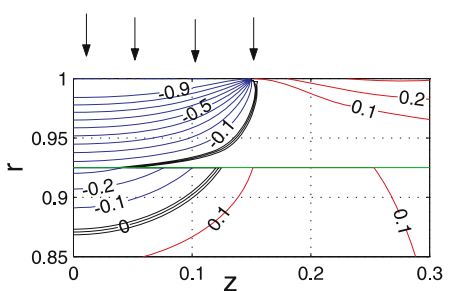

(a) $\sigma_{1} / p_{0}(\phi=0)$

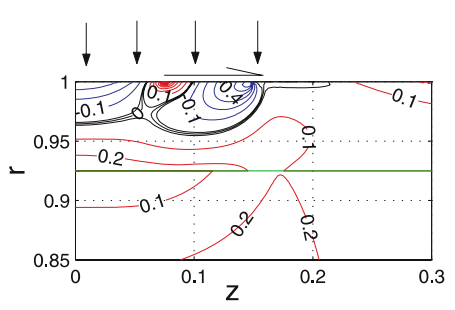

(c) $\sigma_{1} / \mathrm{p}_{0}(\phi=0.5)$

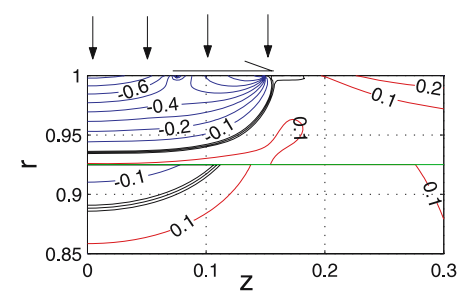

(b) $\sigma_{1} / \mathrm{p}_{0}(\phi=0.3)$

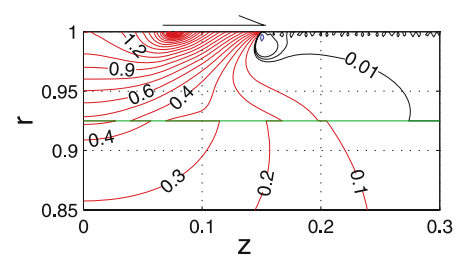

(d) $\sigma_{1} / \mathbf{p}_{0}$ (pure shear)

Figure 18. Contours of $\sigma_{1}$ in cylinder $\left(\mu_{A}=2 \mu_{B}, a=0 \cdot 15 R_{1}\right.$ and $\left.c=0.5 a\right)$.

\subsection{Effect of geometry}

Fellows et al (1995) discussed the effect of finite thickness of the body on the calculation of the contact stresses in the context of fretting. The results presented here provide further insight regarding the tensile stress zones to quantify the severity of fretting. The geometry of component can be cylindrical as in the case of shrink-fit shafts and sleeves rather than strip and half-plane. Hence a comparison among the three geometries is attempted here to explore the geometry-dependent effect on the state of stress near the surface including the interface. The results of three geometries are compared for two different elastic mismatch and two different values of coefficient of friction. Contours of $\tau_{\text {oct }}$ obtained from cylinder are compared with the contours obtained from strip and half-plane (2D plane strain models). Figures 19 and 20 correspond to the cases of soft coating with coefficient of friction $\phi=0.3$ and 0.7 respectively. The figures show that as the coefficient of friction is increased the contours in all the three geometries become more similar. This is due to the fact that for the case of shear loading the stress is concentrated at the surface and hence the actual geometry of the component becomes less important.

Figures 21 and 22 correspond to the case of hard coating with $\phi=0.3$ and 0.7 respectively. Here also we see that as the coefficient of friction increases $\tau_{o c t}$ is more concentrated at the surface.

\subsection{Effect of a $/ R_{1}$ ratio}

Four different values chosen for $a / R_{1}$ ratio are $0.05,0.1,0.3$ and 0.45 with $c=a / 2$ and $R_{2}=0.925 R_{1}$. Since the difference between the cylinder, strip and half-plane depends on the $a / R_{1}$ ratio it is important to study the effect of $a / R_{1}$. Smaller the $a / R_{1}$ ratio better will be the match expected between the strip, cylinder and half-plane. This can be seen from figures 23 and 24 . The results are presented in a window of size $0 \cdot 3 R_{1} \times 0 \cdot 15 R_{1}$. This is because contact width is changing with different values of $a / R_{1}$ ratio. 


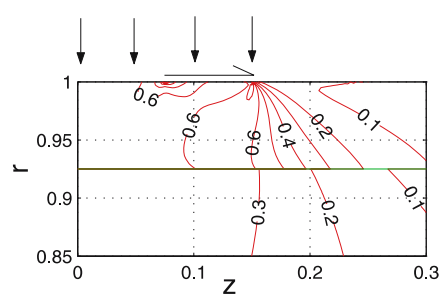

(a) cylinder $\left(\mu_{\mathrm{A}}=0.5 \mu_{\mathrm{B}}, \phi=0.3\right)$

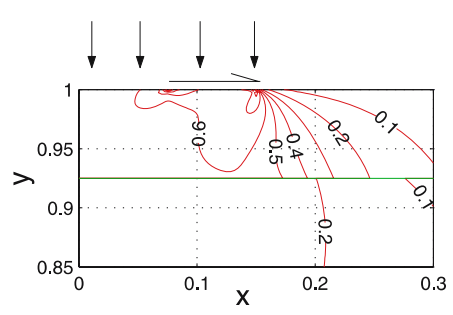

(c) half plane $\left(\mu_{\mathrm{A}}=0.5 \mu_{\mathrm{B}}, \phi=0.3\right)$

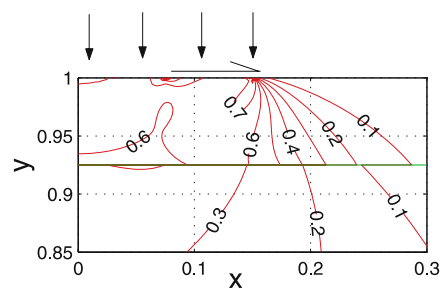

(b) strip $\left(\mu_{\mathrm{A}}=0.5 \mu_{\mathrm{B}}, \phi=0.3\right)$

Figure 19. Contours of $\tau_{\text {oct }}$ (soft coating, low friction with $a=0 \cdot 15 R_{1}$ and $\left.c=0 \cdot 5 a\right)$.

\subsection{Effect of c/a ratio}

The actual nature of contact problem in fretting is generally a mixed boundary problem. The existence of partial slip conditions complicates this situation further. Hence a non-linear way of obtaining the contact parameters $a$ and $c$ is required. However in this work reasonable values of $c / a$ ratio $(0.2,0.4,0.6$ and 0.8$)$ are considered individually for simplification and the effect of the ratio is studied (figures 25 and 26).

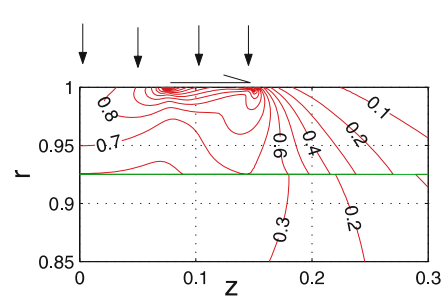

(a) cylinder $\left(\mu_{\mathrm{A}}=0.5 \mu_{\mathrm{B}}, \phi=0.7\right)$

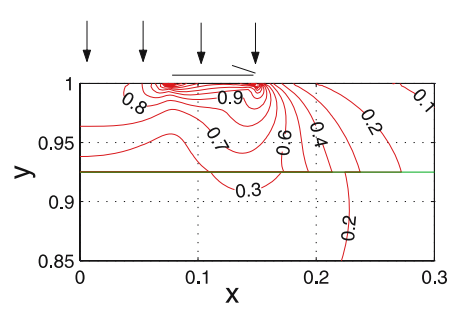

(c) half plane $\left(\mu_{\mathrm{A}}=0.5 \mu_{\mathrm{B}}, \phi=0.7\right)$

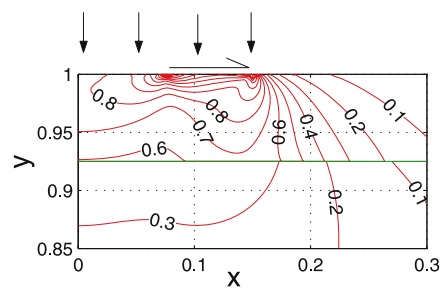

(b) $\operatorname{strip}\left(\mu_{\mathrm{A}}=0.5 \mu_{\mathrm{B}}, \phi=0.7\right)$

Figure 20. Contours of $\tau_{o c t}$ (soft coating, high friction with $a=0.15 R_{1}$ and $c=0.5 a$ ). 


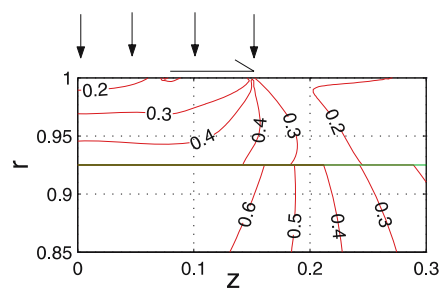

(a) cylinder $\left(\mu_{\mathrm{A}}=2 \mu_{\mathrm{B}}, \phi=0.3\right)$

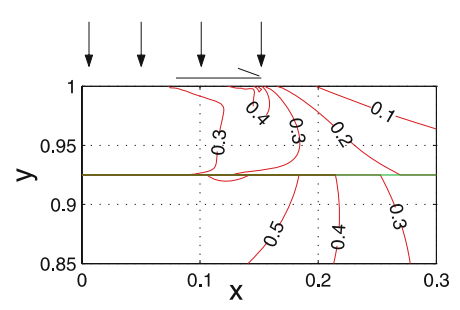

(c) half plane $\left(\mu_{\mathrm{A}}=2 \mu_{\mathrm{B}}, \phi=0.3\right)$

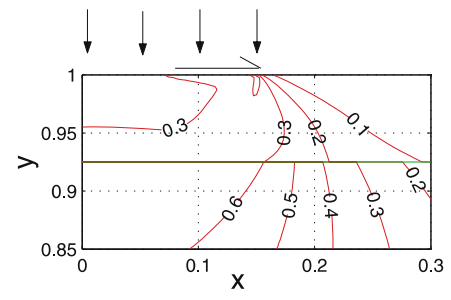

(b) $\operatorname{strip}\left(\mu_{\mathrm{A}}=2 \mu_{\mathrm{B}}, \phi=0.3\right)$

Figure 21. Contours of $\tau_{\text {oct }}$ (hard coating, low friction with $a=0 \cdot 15 R_{1}$ and $c=0 \cdot 5 a$ ).

\subsection{Sign reversal in shear stress}

Fretting tractions may induce subsurface shear stresses of opposite sign. The distance of separation being small which leads to change of sign of $\tau_{r z}$ as one moves from the surface to the depth. This is true for different cases of elastic mismatch (figure 27). This phenomenon is reminiscent of flow reversal in tubes. In this figure it should be noted that window is expanded to cover the entire cylinder radius. Hence for numerical inversion actual values of Bessel functions are used for $\xi=0$ to 350 only and no asymptotic expressions are used.

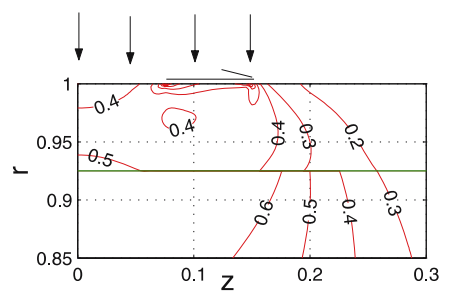

(a) cylinder $\left(\mu_{\mathrm{A}}=2 \mu_{\mathrm{B}}, \phi=0.7\right)$

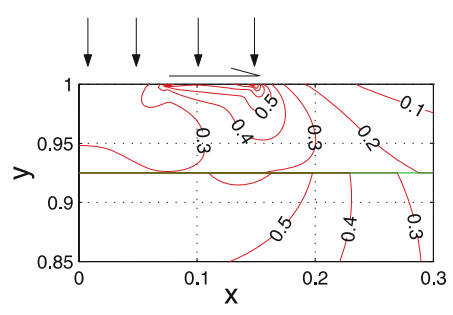

(c) half plane $\left(\mu_{\mathrm{A}}=2 \mu_{\mathrm{B}}, \phi=0.7\right)$

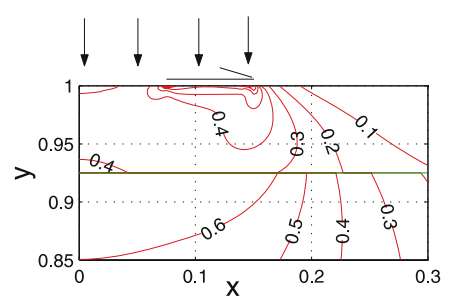

(b) strip $\left(\mu_{\mathrm{A}}=2 \mu_{\mathrm{B}}, \phi=0.7\right)$

Figure 22. Contours of $\tau_{\text {oct }}$ (hard coating, high friction with $a=0.15 R_{1}$ and $c=0.5 a$ ). 


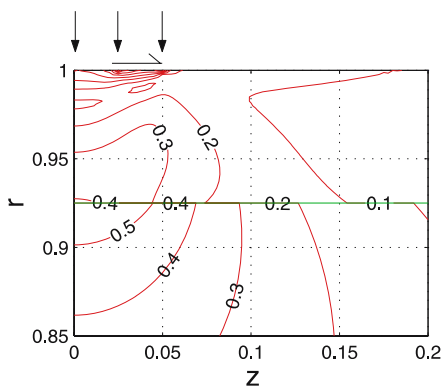

(a) $\tau_{\text {oct }} / \mathrm{p}_{0}\left(\mathrm{a}=0.05 \mathrm{R}_{1}\right)$

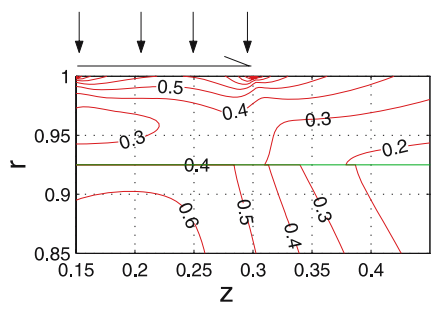

(c) $\tau_{\text {oct }} / \mathrm{p}_{0}\left(\mathrm{a}=0.3 \mathrm{R}_{1}\right)$

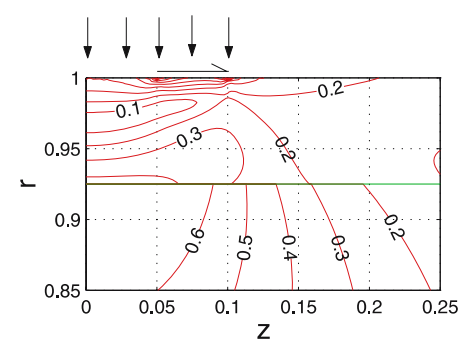

(b) $\tau_{\text {oct }} / \mathrm{p}_{0}\left(\mathrm{a}=0.1 \mathrm{R}_{1}\right)$

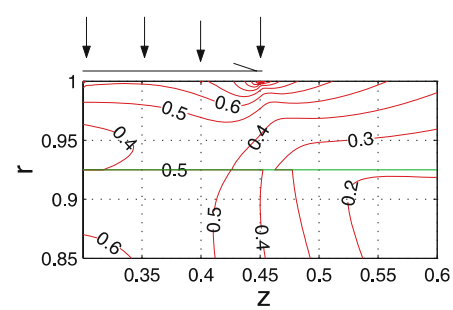

(d) $\tau_{\text {oct }} / p_{0}\left(a=0.45 R_{1}\right)$

Figure 23. Effect of $a / R_{1}\left(c=0.5 a, \mu_{A}=2 \mu_{B}\right.$ and $\left.\phi=0 \cdot 7\right)$.

\subsection{Interface delamination}

It is important to study the tractions along the interface. The ratio of $\tau_{r z} / \sigma_{r}$ exceeding a critical value forms the basis of Mohr-Coulomb theory of failure for brittle materials similar to the

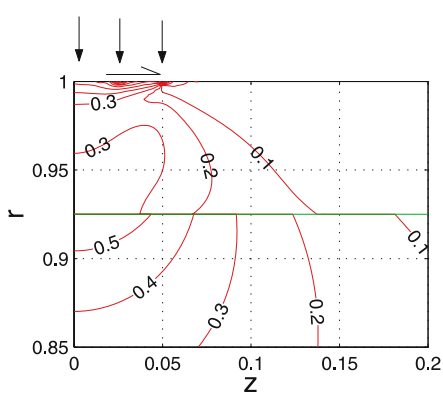

(a) $\tau_{\text {oct }} / \mathrm{p}_{0}(\mathrm{a}=0.05 \mathrm{R} 1)$

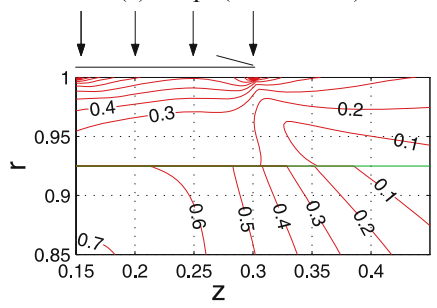

(c) $\tau_{\text {oct }} / \mathrm{p}_{0}(\mathrm{a}=0.3 \mathrm{R} 1)$

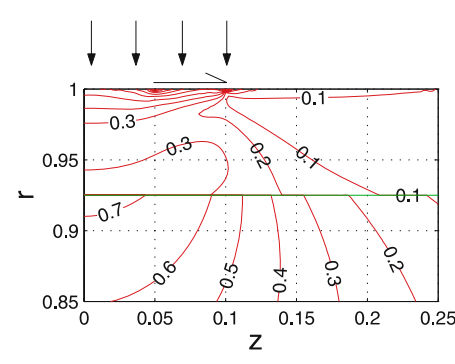

(b) $\tau_{\text {oct }} / \mathrm{p}_{0}(\mathrm{a}=0.1 \mathrm{R} 1)$

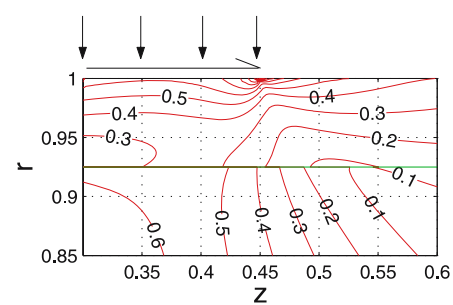

(d) $\tau_{\text {oct }} / \mathrm{p}_{0}(\mathrm{a}=0.45 \mathrm{R} 1)$

Figure 24. Effect of $a / R_{1}$ in strip $\left(c=0.5 a, \mu_{A}=2 \mu_{B}\right.$ and $\left.\phi=0.7\right)$. 


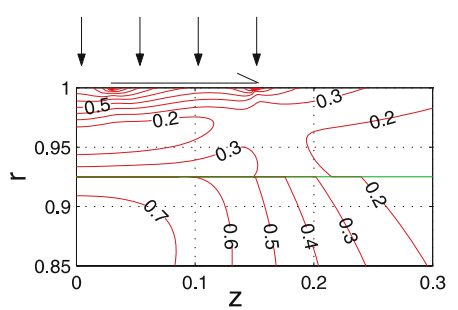

(a) $\tau_{\text {oct }} / \mathrm{p}_{0}(\mathrm{c}=0.2 \mathrm{a})$

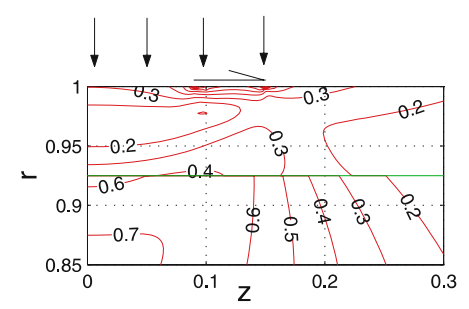

(c) $\tau_{\text {oct }} / p_{0}(\mathrm{c}=0.6 \mathrm{a})$

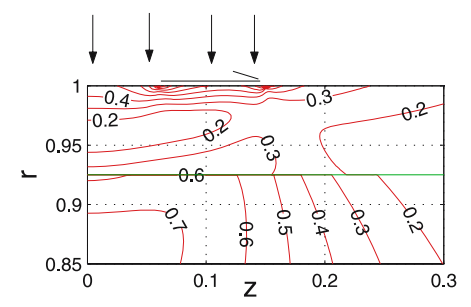

(b) $\tau_{\text {oct }} / p_{0}(c=0.4$ a)

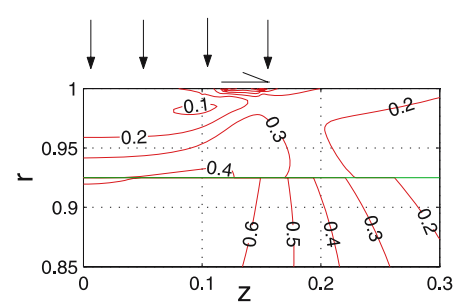

(d) $\tau_{\text {oct }} / \mathrm{p}_{0}(\mathrm{c}=0.8 \mathrm{a})$

Figure 25. Effect of $c / a\left(a=0 \cdot 15 R_{1}, \mu_{A}=2 \mu_{B}\right.$ and $\left.\phi=0 \cdot 7\right)$.

coefficient of friction. A plot of $\tau_{r z} / \sigma_{r}$ ratio along the interface is shown in figure 28 for both hard and soft coating corresponding to both high and low coefficients of friction. The ratio is plotted for three different geometries using three different colours as shown in the legend. It is interesting to note that $\tau / \sigma$ ratio reaches value well over $10 \phi$ as we move away from the loading zone.

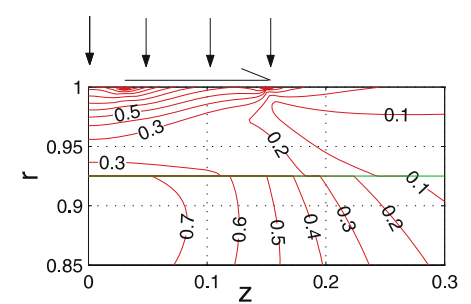

(a) $\tau_{\text {oct }} / p_{0}(\mathrm{c}=0.2 \mathrm{a})$

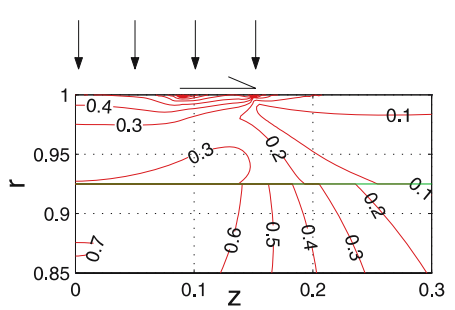

(c) $\tau_{\text {oct }} / p_{0}(\mathrm{c}=0.6 \mathrm{a})$

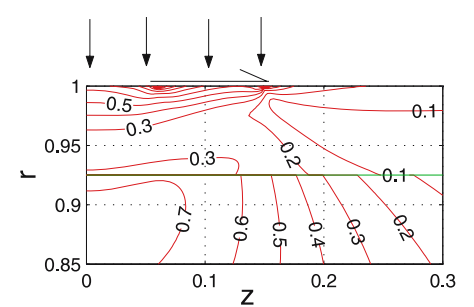

(b) $\tau_{\text {oct }} / \mathrm{p}_{0}(\mathrm{c}=0.4 \mathrm{a})$

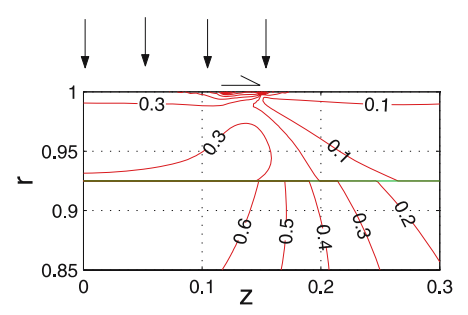

(d) $\tau_{\text {oct }} / \mathrm{p}_{0}(\mathrm{c}=0.8 \mathrm{a})$

Figure 26. Effect of $c / a$ in strip $\left(a=0 \cdot 15 R_{1}, \mu_{A}=2 \mu_{B}\right.$ and $\left.\phi=0 \cdot 7\right)$. 


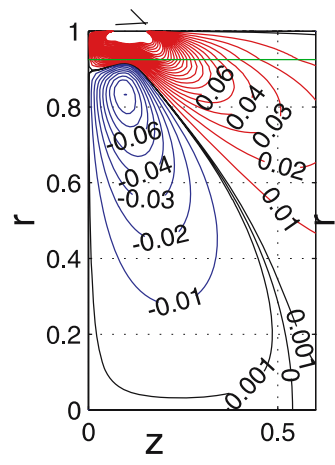

(a) $\mu_{\mathrm{A}}=0.5 \mu_{\mathrm{B}}$

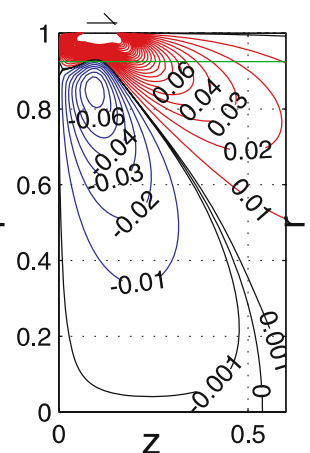

(b) $\mu_{\mathrm{A}}=\mu_{\mathrm{B}}$

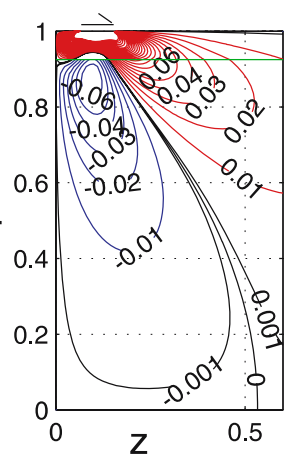

(c) $\mu_{\mathrm{A}}=2 \mu_{\mathrm{B}}$

Figure 27. Shear stress reversal ( $p_{0}=0, a=0 \cdot 15 R_{1}$ and $c=0 \cdot 4 a$ ).

\section{Conclusion}

The effect of varying coating parameters like elastic mismatch between the coating and the substrate, coefficient of friction and the loading geometry on the stresses is discussed in this paper. The main results are:

- Elastic mismatch is important as $\sigma_{z}$ becomes tensile due to bending effect.

- Increase in coefficient of friction leads to concentrated stress in the near surface region.

- The interfacial stress ratio $\tau / \sigma$ is increasing uniformly away from the loading zone reaching values well over $10 \phi$.

- The stresses roughly scale with the elastic shear modulus indicating the continuity of strains across the interface in thin coatings.

(a) $\mu_{\mathrm{A}} / \mu_{\mathrm{B}}=2 \quad \phi=0.3$

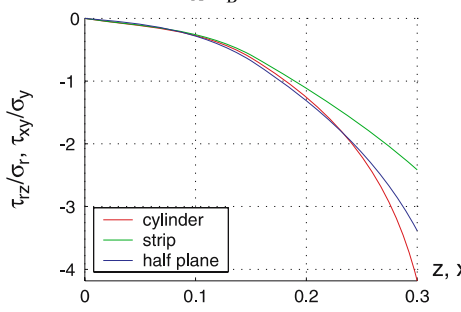

(c) $\mu_{\mathrm{A}} / \mu_{\mathrm{B}}=1 / 2 \quad \phi=0.3$

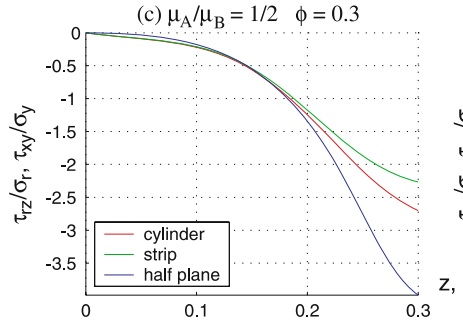

(b) $\mu_{\mathrm{A}} / \mu_{\mathrm{B}}=2 \quad \phi=0.7$

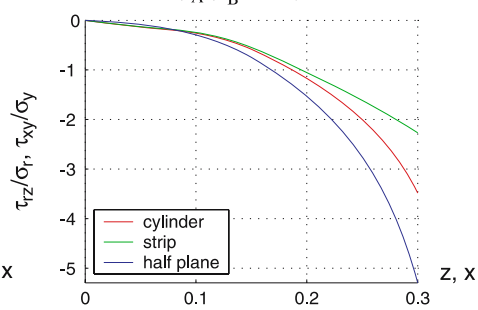

(d) $\mu_{\mathrm{A}} / \mu_{\mathrm{B}}=1 / 2 \quad \phi=0.7$

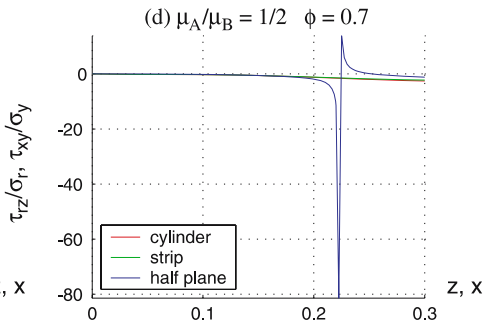

Figure 28. Ratio of $\tau_{r z} / \sigma_{r}$ along interface. 
Based on the results obtained in this paper, we can conclude that coatings of thickness up to $10 \%$ of the cylinder radius may behave like thin shells. Therefore, it is possible to apply shell theory for additional insight particularly concerning bending stresses. It should be noted that shell elements used in FEM provide only an approximate model.

\section{Nomenclature}

$\begin{array}{ll}2 a & \text { Contact width } \\ 2 c & \text { Stick zone width } \\ R_{1} & \text { Radius of cylinder with coating } \\ R_{2} & \text { Radius of cylinder without coating } \\ p_{0} & \text { Applied pressure } \\ q_{0} & \text { Shear developed due to coefficient of friction } \\ P, Q & \text { Pressure and shear tractions along the contact } \\ \phi & \text { Coefficient of friction } \\ \mu & \text { Shear modulus } \\ \nu & \text { Poisson's ratio } \\ E & \text { Average of elastic moduli of coating and substrate } \\ A, B & \text { Represents coating and substrate } \\ r, \theta, z & \text { Cylindrical coordinates } \\ x, y, w & \text { Cartesian coordinates } \\ \Phi & \text { Love/Airy stress function } \\ \sigma_{r}, \sigma_{\theta}, \sigma_{z} & \text { Radial, tangential and axial stresses } \\ \tau_{r z} & \text { Shear stress } \\ \epsilon_{r}, \epsilon_{\theta} \epsilon_{z} & \text { Radial, tangential and axial strains } \\ \gamma_{r z} & \text { Shear strain } \\ u_{r}, u_{z} & \text { Radial and axial displacements } \\ \Delta & \text { Dilatation } \\ \tau_{o c t} & \text { Octahedral shear stress } \\ \xi & \text { Variable of Fourier transform } \\ \eta & \text { Represents } z \text { in cylindrical coordinates } \\ \nabla^{2} & \quad \text { and } x \text { in Cartesian coordinates } \\ I_{0}, I_{1} & \text { Laplace's operator } \\ K_{0}, K_{1} & \text { Modified Bessel functions of first kind } \\ & \text { Modified Bessel functions of second kind }\end{array}$

Appendix A. Expressions for $a_{i j}$ and $b_{i j}$

Appendix A.1 Cylinder

$$
\begin{aligned}
& a_{11}=-I_{1}(\xi r) / I_{0}\left(\xi R_{1}\right) / \xi \\
& a_{12}=K_{1}(\xi r) / K_{0}\left(\xi R_{1}\right) / \xi \\
& a_{13}=-\xi r I_{0}(\xi r) / I_{0}\left(\xi R_{1}\right) / \xi \\
& a_{14}=\xi r K_{0}(\xi r) / K_{0}\left(\xi R_{1}\right) / \xi
\end{aligned}
$$




$$
\begin{aligned}
& a_{21}=I_{0}(\xi r) / I_{0}\left(\xi R_{1}\right) / \xi \\
& a_{22}=K_{0}(\xi r) / K_{0}\left(\xi R_{1}\right) / \xi \\
& a_{23}=\left[4(1-v) I_{0}(\xi r)+\xi r I_{1}(\xi r)\right] / I_{0}\left(\xi R_{1}\right) / \xi \\
& a_{24}=\left[-4(1-v) K_{0}(\xi r)+\xi r K_{1}(\xi r)\right] / K_{0}\left(\xi R_{1}\right) / \xi \\
& a_{31}=\left[-I_{0}(\xi r)+\frac{I_{1}(\xi r)}{\xi r}\right] / I_{0}\left(\xi R_{1}\right) \\
& a_{32}=\left[-K_{0}(\xi r)-\frac{K_{1}(\xi r)}{\xi r}\right] / K_{0}\left(\xi R_{1}\right) \\
& a_{33}=\left[-(1-2 v) I_{0}(\xi r)-\xi_{r} I_{1}(\xi r)\right] / I_{0}\left(\xi R_{1}\right) \\
& a_{34}=\left[(1-2 v) K_{0}(\xi r)-\xi_{r} K_{1}(\xi r)\right] / K_{0}\left(\xi R_{1}\right) \\
& a_{41}=I_{1}(\xi r) / I_{0}\left(\xi R_{1}\right) \\
& a_{42}=-K_{1}(\xi r) / K_{0}\left(\xi R_{1}\right) \\
& a_{43}=\left[2(1-v) I_{1}(\xi r)+\xi r I_{0}(\xi r)\right] / I_{0}\left(\xi R_{1}\right) \\
& a_{44}=\left[2(1-v) K_{1}(\xi r)-\xi r K_{0}(\xi r)\right] / K_{0}\left(\xi R_{1}\right) \\
& a_{51}=\frac{-I_{1}(\xi r)}{\xi r} / I_{0}\left(\xi R_{1}\right) \\
& a_{52}=\frac{K_{1}(\xi r)}{\xi r} / K_{0}\left(\xi R_{1}\right) \\
& a_{53}=-(1-2 \nu) I_{0}\left(\xi_{r}\right) / I_{0}\left(\xi R_{1}\right) \\
& a_{54}=(1-2 v) K_{0}\left(\xi_{r}\right) / K_{0}\left(\xi R_{1}\right) \\
& a_{61}=I_{0}(\xi r) / I_{0}\left(\xi R_{1}\right) \\
& a_{62}=K_{0}(\xi r) / K_{0}\left(\xi R_{1}\right) \\
& a_{63}=\left[2(2-v) I_{0}(\xi r)+\xi r I_{1}(\xi r)\right] / I_{0}\left(\xi R_{1}\right) \\
& a_{64}=\left[-2(2-v) K_{0}(\xi r)+\xi r K_{1}(\xi r)\right] / K_{0}\left(\xi R_{1}\right) \\
& b_{11}=-I_{1}(\xi r) / I_{0}\left(\xi R_{1}\right) / \xi \\
& b_{13}=-\xi r I_{0}(\xi r) / I_{0}\left(\xi R_{1}\right) / \xi
\end{aligned}
$$




$$
\begin{aligned}
& b_{21}=I_{0}(\xi r) / I_{0}\left(\xi R_{1}\right) / \xi \\
& b_{23}=\left[4(1-v) I_{0}(\xi r)+\xi r I_{1}(\xi r)\right] / I_{0}\left(\xi R_{1}\right) / \xi \\
& b_{31}=\left[-I_{0}(\xi r)+\frac{I_{1}(\xi r)}{\xi r}\right] / I_{0}\left(\xi R_{1}\right) \\
& b_{33}=\left[-(1-2 v) I_{0}(\xi r)-\xi r I_{1}(\xi r)\right] / I_{0}\left(\xi R_{1}\right) \\
& b_{41}=I_{1}(\xi r) / I_{0}\left(\xi R_{1}\right) \\
& b_{43}=\left[2(1-v) I_{1}(\xi r)+\xi r I_{0}(\xi r)\right] / I_{0}\left(\xi R_{1}\right) \\
& b_{51}=\frac{-I_{1}(\xi r)}{\xi r} / I_{0}\left(\xi R_{1}\right) \\
& b_{53}=-(1-2 v) I_{0}(\xi r) / I_{0}\left(\xi R_{1}\right) \\
& b_{61}=I_{0}(\xi r) / I_{0}\left(\xi R_{1}\right) \\
& b_{63}=\left[2(2-v) I_{0}(\xi r)+\xi r I_{1}(\xi r)\right] / I_{0}\left(\xi R_{1}\right)
\end{aligned}
$$

Appendix A.2 Strip

$$
\begin{aligned}
& a_{11}=e^{-\xi\left(y-Y_{1}\right)} / \xi \\
& a_{12}=-e^{\xi\left(y-Y_{1}\right)} / \xi \\
& a_{13}=[\xi y+(1-2 v)] e^{-\xi\left(y-Y_{1}\right)} / \xi \\
& a_{14}=[-\xi y+(1-2 v)] e^{\xi\left(y-Y_{1}\right)} / \xi \\
& a_{21}=e^{-\xi\left(y-Y_{1}\right)} / \xi \\
& a_{22}=e^{\xi\left(y-Y_{1}\right)} / \xi \\
& a_{23}=[\xi y-2(1-v)] e^{-\xi\left(y-Y_{1}\right)} / \xi \\
& a_{24}=[\xi y+2(1-v)] e^{\xi\left(y-Y_{1}\right)} / \xi \\
& a_{31}=-e^{-\xi\left(y-Y_{1}\right)} \\
& a_{32}=-e^{\xi\left(y-Y_{1}\right)}
\end{aligned}
$$




$$
\begin{aligned}
& a_{33}=-\xi y e^{-\xi\left(y-Y_{1}\right)} \\
& a_{34}=-\xi y e^{\xi\left(y-Y_{1}\right)} \\
& a_{41}=-e^{-\xi\left(y-Y_{1}\right)} \\
& a_{42}=e^{\xi\left(y-Y_{1}\right)} \\
& a_{43}=(1-\xi y) e^{-\xi\left(y-Y_{1}\right)} \\
& a_{44}=(1+\xi y) e^{\xi\left(y-Y_{1}\right)} \\
& a_{51}=0 \\
& a_{52}=0 \\
& a_{53}=-2 v e^{-\xi\left(y-Y_{1}\right)} \\
& a_{54}=2 v e^{\xi\left(y-Y_{1}\right)} \\
& a_{61}=e^{-\xi\left(y-Y_{1}\right)} \\
& a_{62}=e^{\xi\left(y-Y_{1}\right)} \\
& a_{63}=(\xi y-2) e^{-\xi\left(y-Y_{1}\right)} \\
& a_{64}=(\xi y+2) e^{\xi\left(y-Y_{1}\right)} \\
& b_{11}=-\sinh (\xi y) / e^{\xi Y_{1}} / \xi \\
& b_{13}=-[\xi y \cosh (\xi y)-(1-2 v) \sinh (\xi y)] / e^{\xi Y_{1}} / \xi \\
& b_{21}=\cosh (\xi y) / e^{\xi Y_{1}} / \xi \\
& b_{23}=[\xi y \sinh (\xi y)+2(1-v) \cosh (\xi y)] / e^{\xi Y_{1}} / \xi \\
& b_{31}=-\cosh (\xi y) / e^{\xi Y_{1}} \\
& b_{33}=-\xi y \sinh (\xi y) / e^{\xi Y_{1}} \\
& b_{41}=\sinh (\xi y) / e^{\xi Y_{1}} \\
& b_{43}=[\xi y \cosh (\xi y)+\sinh (\xi y)] / e^{\xi Y_{1}}
\end{aligned}
$$




$$
\begin{aligned}
& b_{51}=0 \\
& b_{53}=2 v \cosh (\xi y) / e^{\xi Y_{1}} \\
& b_{61}=\cosh (\xi y) / e^{\xi Y_{1}} \\
& b_{63}=[\xi y \sinh (\xi y)+2 \cosh (\xi y)] / e^{\xi Y_{1}}
\end{aligned}
$$

Appendix A.3 Half-plane

$$
\begin{aligned}
& a_{11}=e^{-\xi y} / \xi \\
& a_{12}=-e^{\xi y} / \xi \\
& a_{13}=(1-2 v+\xi y) e^{-\xi y} / \xi \\
& a_{14}=(1-2 v-\xi y) e^{\xi y} / \xi \\
& a_{21}=e^{-\xi y} / \xi \\
& a_{22}=e^{\xi y} / \xi \\
& a_{23}=[\xi y-2(1-v)] e^{-\xi y} / \xi \\
& a_{24}=[\xi y+2(1-v)] e^{\xi y} / \xi \\
& a_{31}=-e^{-\xi y} \\
& a_{32}=-e^{\xi y} \\
& a_{33}=-\xi y e^{-\xi y} \\
& a_{34}=-\xi y e^{\xi y} \\
& a_{41}=-e^{-\xi y} \\
& a_{42}=e^{\xi y} \\
& a_{43}=(1-\xi y) e^{-\xi y} \\
& a_{44}=(1+\xi y) e^{\xi y} \\
& a_{51}=0 \\
& a_{52}=0 \\
& =
\end{aligned}
$$




$$
\begin{aligned}
& a_{53}=-2 v e^{-\xi y} \\
& a_{54}=2 v e^{\xi y} \\
& a_{61}=e^{-\xi y} \\
& a_{62}=e^{\xi y} \\
& a_{63}=(\xi y-2) e^{-\xi y} \\
& a_{64}=(\xi y+2) e^{\xi y} \\
& b_{11}=e^{-\xi y} / \xi \\
& b_{13}=(1-2 v+\xi y) e^{-\xi y} / \xi \\
& b_{21}=e^{-\xi y} / \xi \\
& b_{23}=[\xi y-2(1-v)] e^{-\xi y} / \xi \\
& b_{31}=-e^{-\xi y} \\
& b_{33}=-\xi y e^{-\xi y} \\
& b_{41}=-e^{-\xi y} \\
& b_{43}=(1-\xi y) e^{-\xi y} \\
& b_{51}=0 \\
& b_{53}=-2 v e^{-\xi y} \\
& b_{61}=e^{-\xi y} \\
& b_{63}=-(2-\xi y) e^{-\xi y} \\
& =15
\end{aligned}
$$

\section{Appendix B. Scaling factors}

\begin{tabular}{lcccc}
\hline & \multicolumn{2}{c}{ soft coating } & \multicolumn{2}{c}{ hard coating } \\
\cline { 2 - 5 } & stress & strain & stress & strain \\
\hline $\begin{array}{l}\text { coating } \\
\text { substrate }\end{array}$ & $\mu_{B} / \mu_{A}$ & 1 & 1 & $\mu_{A} / \mu_{B}$ \\
\hline
\end{tabular}




\section{References}

Ciavarella M, Demelio G 2001 A review of analytical aspects of fretting fatigue, with extension to damage parameters, and application to dovetail joints. Int. J. Solids and Struct. 38: 1791-1811

Dobromirsky J, Smith I 1986 A stress analysis of a shaft with a press-fitted hub subjected to cyclic axial loading. Int. J. Mech. Sci. 28(1): 41-52

Erdogan F, Ozbek T 1969 Stresses in fibre-reinfored composites with imperfect bonding. J. Appl. Mech. 865-869

Farris T N, Kokini K, Demir I 1989 The cylindrical crack. J. Appl. Mech. 56: 981-983

Farris T N, Murthy H, Matlik J F 2003 Fretting Fatigue, In R O Rithcie and Y Murakami, eds., Comprehensive structural integrity Elsevier, Pergamon Press 4: 281-326

Fellows L, Nowell D, Hills D A 1995 Contact stresses in a moderately thin strip (with particular reference to fretting experiments). Wear 185: 235-238

Golden P J, Hutson A, Sundaram V, Arps J H 2007 Effect of surface treatments on fretting fatigue of Ti-6Al-4V. Int. J. Fatigue 29: 1302-1310

Goncalves C A, Araujo J A, Mamiya E N 2005 Multiaxial fatigue: A stress based criterion for hard metals. Int. J. Fatigue 27: 177-187

Hager C H, Sanders J H, Sharma S 2004 Characterization of mixed and gross slip fretting wear regimes in Ti-6Al-4V interfaces at room temperature. Wear 257: 167-180

Itou S 1990 Stresses around a cylindrical interface crack under shear. Eng. Fracture Mech. 36(4): $631-638$

Nishida T, Mizutani J, Mutoh Y, Maejima M 2003 Effect of lubricating anodic film on fretting fatigue strength of aluminium alloy. Fretting fatigue: Advances in basic understaning and applications, ASTM STP 1425

Nowell D, Araujo J A 2003 Application of multiaxial fatigue parameters to fretting contacts with high stress gradients. Fretting fatigue: Advances in basic understaning and applications, ASTM STP 1425

Okane M, Shiozawa K, Hiki M, Suzuki K 2003 Fretting fatigue properties of WC-Co thermal sprayed nicrmo steel. Fretting fatigue: Advances in basic understaning and applications, ASTM STP 1425

Ozbek T, Erdogan F 1969 Some elasticity problems in fibre-reinforced composites with imperfect bonds. Int. J. Eng. Sci. 7: 931-946

Papadopoulos IV, Davoli P, Gorla C, Filippini M, Bernasconi A 1997 A comparative study of multiaxial high-cycle fatigue criteria for metals. Int. J. Fatigue 19: 219-235

Rajasekaran B, Raman S G S, Joshi S V, Sundararajan G 2008 Performance of plasma sprayed and detonation gun sprayed $\mathrm{Cu}-\mathrm{Ni}-\mathrm{In}$ coatings on Ti-6Al-4V under plain fatigue and fretting fatigue loading. Mater. Sci. and Eng. A 479(1-2): 83-92

Timoshenko S, Goodier J 1970 Theory of Elasticity. Engineering Mechanics Series (Mineola, NewYork: McGraw-Hill International Editions)

Williams J 2005 Engineering Tribology. Cambridge University Press

Xu J, Zhou Z R, Zhang C H, Zhu M H, Luo J 2007 An investigation of fretting wear behaviours of bonded solid lubricant coatings. J. Mater. Proc. Technol. 182: 146-151 